Internal Report

DESY M 04-02

March 2005

\title{
Emittance Preservation in Linear Accelerators
}

\author{
M. Minty
}

Deutsches Elektronen-Synchrotron DESY, Hamburg 


\title{
Emittance Preservation in Linear Accelerators
}

\author{
M. Minty
}

\begin{abstract}
.
In linear colliders preservation of the phase space density of charged particles during acceleration to high energies is essential. In practice, the electromagnetic fields which govern the beam transport may not be sufficiently well understood. This may arise, for example, from magnet and structure alignment and/or manufacturing errors, timevarying electromagnetic fields due to component vibration or imperfect regulation, or at high beam currents, from beam-induced fields. These inadequacies may be overcome using measurements of the beam response. In this report we review such methods for preserving single-bunch beam emittances with experimental results from the Stanford Linear Collider.
\end{abstract}

\section{INTRODUCTION}

Minimizing dilutions to the beam's phase space volume, or emittance, over extended periods is vital for ensuring the highest possible luminosity at colliders. The Stanford Linear Collider (SLC) is the first high-energy linear collider and much practical experience on emittance preservation comes from operating this accelerator. Since considerable understanding of beam dynamics in linear colliders was motivated, stimulated, and triggered by observations from the SLC, it may be helpful if one has been made familiar with this accelerator. For this a brief overview of the SLC is given.

The geometry of the SLC is shown in Fig. 1. The $3 \mathrm{~km}$ linac accelerates three bunches simultaneously - a positron followed by two electron bunches. The constant gradient structures are driven by klystrons at $2856 \mathrm{MHz}$ with a $120 \mathrm{~Hz}$ repetition frequency. Typical beam parameters include the bunch populations of $4 \times 10^{10}$ particles, the bunch lengths $\sigma_{z} \sim 1 \mathrm{~mm}$, the energy spreads $\delta<0.1 \%$, and normalized transverse emittances of $\gamma \epsilon_{x}=(4-5) \times 10^{-5}$ m-rad horizontally and $\gamma \epsilon_{y}=(0.5-1.0) \times 10^{-5} \mathrm{~m}$-rad vertically measured at the end of the linac at 50 $\mathrm{GeV}$. 


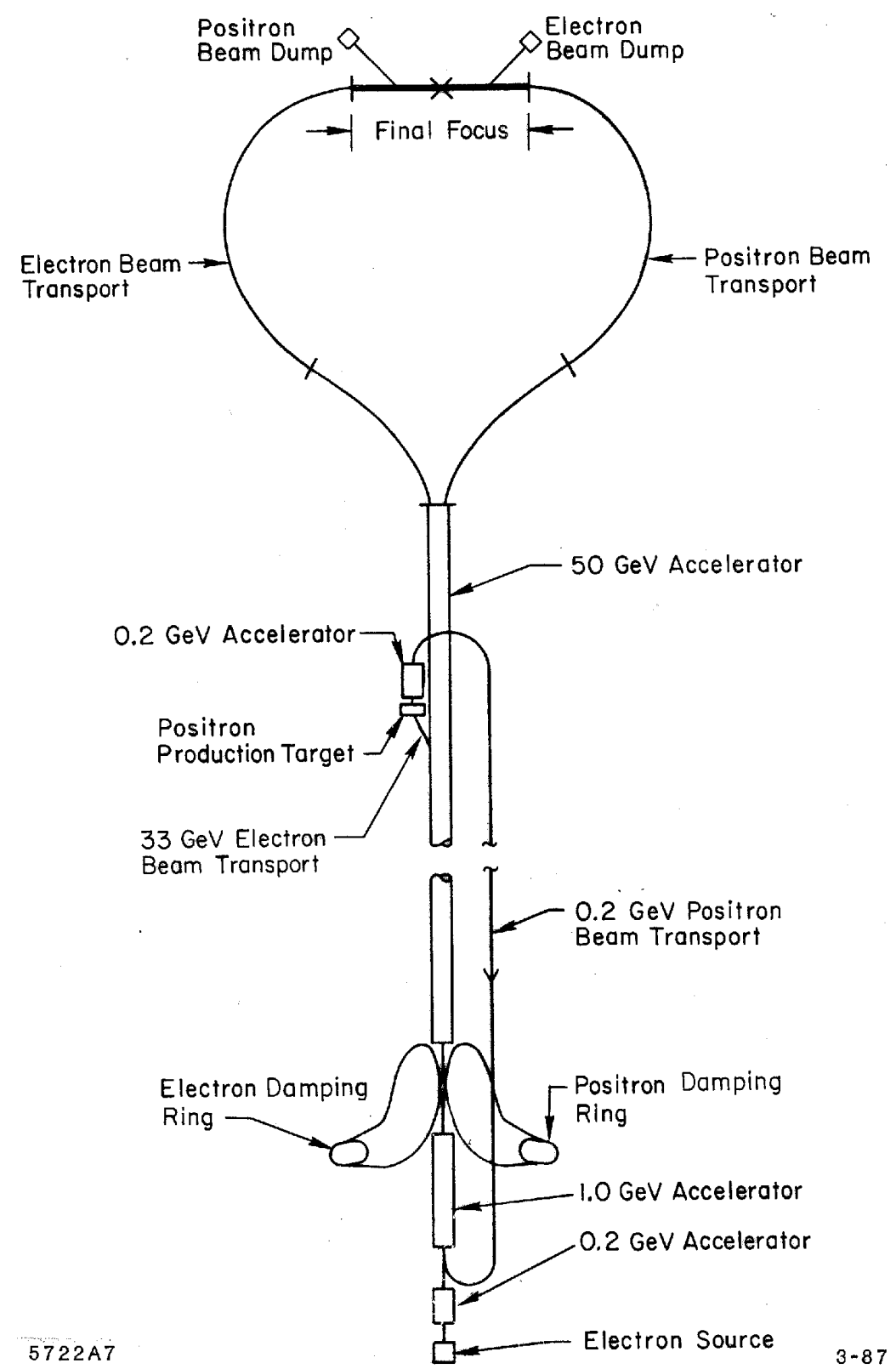

FIGURE 1. Overview of the SLC. Two electron bunches are produced at the source, accelerated to $1.2 \mathrm{GeV}$, and injected into the electron damping ring where they are damped by emission of synchrotron radiation and rf acceleration. The two bunches are then extracted, compressed, and then accelerated to high energy in the main linac. The leading electron bunch is extracted at 20 $\mathrm{GeV}$ and deflected onto to the positron production target. The trailing bunch is accelerated to $50 \mathrm{GeV}$ and transported to the interaction point through the collider arc and final focus. The positron beam is transported at low energy through the beam transport line, accelerated to 1.2 $\mathrm{GeV}$, and radiation damped in the positron damping ring. The positron bunch is then extracted, compressed, accelerated to $50 \mathrm{GeV}$ (leading the 2 electron bunches), transported through the arc, and brought into collision with the electrons at the interaction point 
Shown in Fig. 2 are measurements [1] of the normalized horizontal emittance made after several years of SLC operation measured at various locations along the collider as a function of bunch charge. By this time many emittance enlargement effects had been reduced significantly such as optical mismatches in the ring-tolinac transport line [2-4], dilutions arising from quadrupole and accelerator misalignments [5-9], and coupling generated in the nonplanar collider arcs [10]. In addition, both long and short term variations in the beam properties at injection [11] and in the main linac were controlled using orbit feedback [12-20] and BNS damping. At injection, fluctuations and drift of the transverse beam position and angle were regulated using launch feedback loops $[13,14]$ while the injection phase was held nominally constant by maintaining the phase of the injected beam using analog feedback between the rf systems of the linac and damping ring [21].

From Fig. 2, emittance growth in the ring-to-linac transport line was dominated by chromatic effects [22] which were exaggerated by high-current bunch lengthening in the damping rings [23-29] and bunch compression. In the main linac the emittance dilutions were governed by both wakefield and chromatic effects which became increasingly important at high beam currents [8,22,30-33]. Minimizing and stabilizing such current-dependent dilutions proved essential for achieving routine, high-luminosity operation. By the end of the SLC program, the emittance growth from the (uncoupled) damping rings to the end of the linac was routinely maintained to less than (15-20)\% at bunch charges of $4 \times 10^{10}$ both horizontally and vertically compared with nearly a factor of 3 increase seen in Fig. 2.

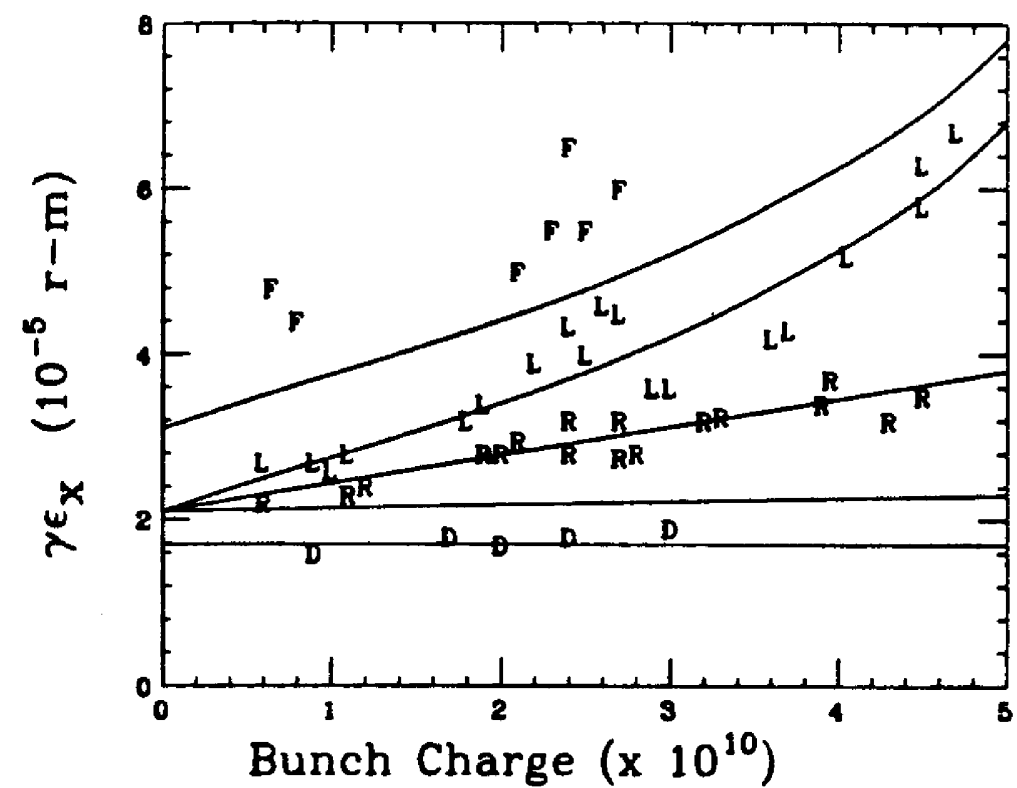

\section{Radiation in arcs}

Linac wakefield and chromatic effects

Ring-to-linac chromatic effects

\section{Ring short store}

Ring equilibrium emittance

FIGURE 2. Emittance growth from the SLC linac entrance to the final focus as a function of bunch population. Measurement locations are denoted by $\mathrm{D}$ at the exit to the damping ring, $\mathrm{R}$ at the linac entrance, $\mathrm{L}$ at the end of the linac, and $\mathrm{F}$ in the final focus. Courtesy J. Seeman (2000). 
In this report emittance preservation during acceleration in the linac proper will be discussed. In sections 2 and 3 basic concepts in transverse and longitudinal emittance preservation, respectively, will be reviewed. In section 4 will be described commonly applied steering algorithms. A conclusion is given in section 5 . In appendix $\mathrm{A}$ the emittance is defined. In appendix B emittance measurement procedures are described.

\section{TRANSVERSE EMITTANCE PRESERVATION}

The transverse dynamics of a single particle in a linac is governed by three variables: the transverse wakefield $W_{\perp}$, acceleration $\frac{d E}{d s}$, and $k$ the lattice focussing. In the following we will consider special cases of the general equation of motion $[34,35]$ given by ${ }^{1}$

$$
\frac{d}{d s}\left[E(s) \frac{d x(z, s)}{d s}\right]+E(s) k^{2}(s) x(z, s)=e^{2} \int_{z}^{\infty} \rho\left(z^{\prime}\right) W_{\perp}\left(z^{\prime}-z\right) x\left(z^{\prime}, s\right) d z^{\prime},
$$

where $s$ represents the longitudinal coordinate and $z$ gives the longitudinal coordinate relative to the bunch center. Here $\rho(z)$ is the longitudinal distribution function of the beam which, in the approximation of zero transverse dimension as in reference [34], is equal to the line density of particles in the bunch. This approximation is valid provided that the beam sizes are much less than that of the vacuum chamber so that the transverse wakefield may be taken to be uniform across the bunch's transverse dimensions. For a particle at position $z$ the transverse wakefield is evaluated over the preceeding particles only. In the special cases to be discussed below, the lattice strength $k(s)$ will be assumed to be smoothly varying rather than consisting of discrete quadrupoles.

Case i: $W_{\perp}=0$ - zero current limit

$$
\begin{aligned}
E & =E_{0}, \text { the beam energy at injection, or } \frac{d E}{d s}=0 \text { - no acceleration } \\
k & =k_{0} \text { - constant gradient }
\end{aligned}
$$

In this case with initial conditions at injection $x(0)=\hat{x}$ and $x^{\prime}(0)=0$,

$$
\frac{d^{2} x}{d s^{2}}+k_{0}^{2} x=0
$$

with solution $x(s)=\hat{x} \cos k_{0} s$ which represents betatron oscillations of peak amplitude $\hat{x}$ about a reference trajectory $x_{c}+x_{\eta}$. In general, the deviation of the particle trajectory is given by

1) the original notation of refs. [34] and [35] has been modified slightly noting

$$
\gamma(s)=\frac{E(s)}{m c^{2}} \text { and } k(s)=\frac{2 \pi}{\lambda(s)},
$$

where $\gamma$ is the Lorentz factor, $E$ is the beam energy, $m c^{2}$ is the particle rest mass, $\lambda(s)$ is the instantaneous wavelength of betatron focusing, and $e^{2}=r_{0} m c^{2}$, where $r_{0}$ is the classical electron radius. 


$$
\begin{aligned}
x & =x_{c}+x_{\beta}+x_{\eta} \\
& =x_{c}+x_{\beta}+\eta \delta .
\end{aligned}
$$

For notational simplicity, the solutions for $x$ in Eq. 3 as in the remainder of this chapter will refer to $x_{\beta}$ (the subscript will be omitted). In Eq. $4 x_{c}$ represents the central trajectory which is defined as the mean orbit that an on-energy particle would follow through the lattice. Ideally this term is zero if the orbit is flat (for a planar linac) passing through perfectly aligned structures and magnets. Due to misalignments $x_{c}$ is in practice not perfectly linear. The term $x_{\eta}=\eta \delta$ gives the additive contribution arising from an energy deviation of the particle, where $\eta$ is the dispersion, and $\delta$ is the relative energy deviation of the particle from that of the design particle.

Case ii: $W_{\perp}=0$ - zero current limit

$E=E_{0}(1+G \cdot s)$ - linear acceleration with gradient $G$

$k=k_{0}$ - constant gradient

The equation of motion is

$$
\frac{d^{2} x}{d s^{2}}+\frac{1}{E}\left(\frac{d E}{d s}\right) \frac{d x}{d s}+k_{0}^{2} x=0
$$

with solution for $x(0)=\hat{x}$ and $x^{\prime}(0)=0$

$$
x(s)=\hat{x} \sqrt{\frac{E_{0}}{E(s)}} \cos k_{0} s .
$$

This result shows that the betatron oscillations damp as $\frac{1}{\sqrt{E}}$.

Damping of the beam's transverse dimensions in a linear accelerator is conceptually easy to visualize. Sketched in Fig. 3a is the particle momentum at energy $E_{0}$ decomposed vectorially into its transverse momentum $p_{\perp}$ and longitudinal momentum $p_{\|}$. The angle $x^{\prime}$ is

$$
x^{\prime}=\tan ^{-1}\left(\frac{p_{\perp}}{p_{\|}}\right) \approx \frac{p_{\perp}}{p_{\|}} \text {for } p_{\perp}<<p_{\|}
$$

In Fig. 3b the decomposed momentum after acceleration by $p_{\|}{ }^{\prime} \Delta s$ is shown. The particles' transverse momentum $p_{\perp}$ is unchanged by the acceleration and

$$
\begin{aligned}
x^{\prime}(s+\Delta s) & \approx \frac{p_{\perp}}{p_{\|}\left(1+\frac{p_{\|}^{\prime}}{p_{\|}} \Delta s\right)} \approx \frac{p_{\perp}}{p_{\|}}\left(1-\frac{p_{\|}^{\prime}}{p_{\|}} \Delta s\right) \\
& \approx x^{\prime}(s)\left(1-\frac{p_{\|}^{\prime}}{p_{\|}} \Delta s\right) .
\end{aligned}
$$

Differentiating gives 


$$
\begin{aligned}
x^{\prime \prime}(s) & \equiv \frac{x^{\prime}(s+\Delta s)-x^{\prime}(s)}{\Delta s} \approx-\frac{p_{\|}^{\prime}}{p_{\|}} x^{\prime}(s) \text { or } \\
x^{\prime \prime} & +\frac{E^{\prime}(s)}{E_{0}} x^{\prime}(s)=0
\end{aligned}
$$

which is Eq. 5 with $k=0$.
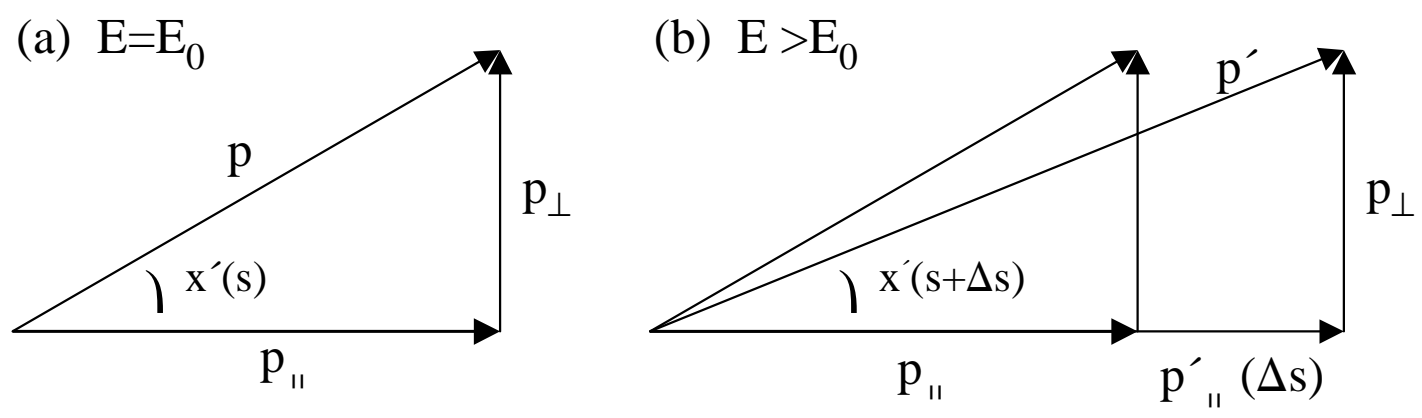

FIGURE 3. Illustration of damping of transverse oscillations in linear accelerators.

From appendix A the final emittance scales as

$$
\epsilon \sim<x^{2}>^{\frac{1}{2}} \sim \frac{E_{0}}{E} \epsilon_{0}=\frac{\gamma_{0}}{\gamma} \epsilon_{0}
$$

where $\gamma$ is again the Lorentz factor. That is, the emittance 'damps' as $\frac{1}{\gamma}$. For this reason, in practice one often expresses emittances as $\gamma \epsilon$ along the linac as it is this quantity which is conserved in the absence of dissipative forces.

So far the transverse motion of only a single particle has been considered. For a bunch consisting of multiple particles, the situation is more complicated since particles of different energy are focussed differently. The lattice focussing depends on the beam energy as

$$
k^{2}=\frac{e c}{E \beta} \frac{\partial B_{z}}{\partial x}
$$

where $\beta \gamma=\sqrt{\gamma^{2}-1}$ and $\frac{\partial B_{z}}{\partial x}$ describes the quadrupole magnetic fields. The motion of the bunch centroid (defined as the position of the mean of the bunch charge distribution) after a net displacement of the bunch may therefore not be damped as $\frac{1}{\sqrt{E}}$. That is, a macroparticle approximation for centroid motion breaks down if the bunch has an internal energy spread.

The measured horizontal beam centroid motion is shown as a function of position along the SLC linac $[1,8]$ in Fig. 4 for different bunch populations. At low current the centroid motion decays faster than as $\frac{1}{\sqrt{E}}$. This is due to the bunch energy spread which causes a spread in the phase advance of the particles within the bunch. At $2.5 \times 10^{10}$ particles the longitudinal profile had been optimized and the 
centroid motion is seen to decay at least initially as $\frac{1}{\sqrt{E}}$ like that of a single particle. Towards the end of the linac and at higher bunch charge, the transverse dynamics is more complicated and $W_{\perp} \neq 0$ must be considered.

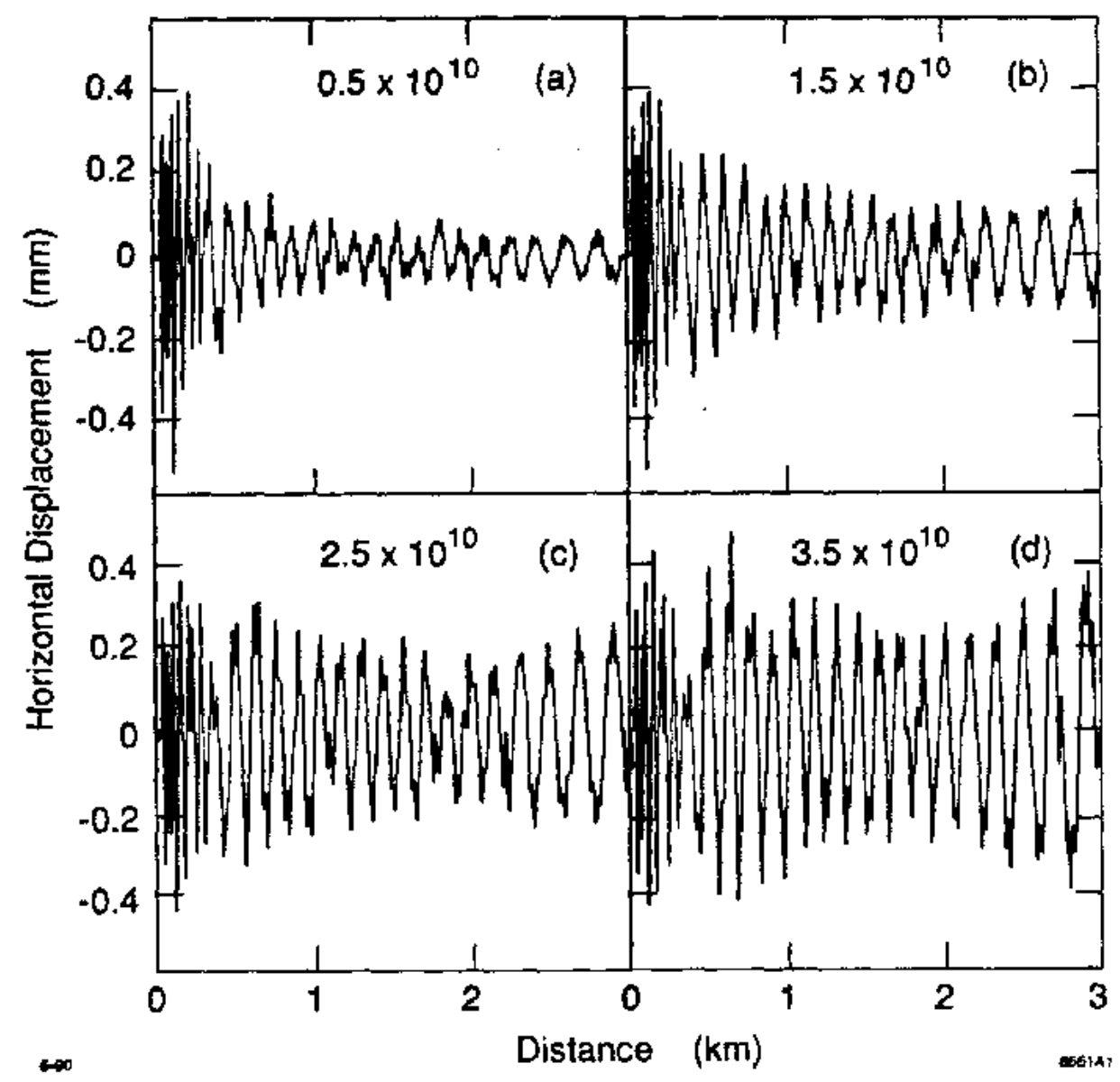

FIGURE 4. Measured horizontal trajectories versus charge at the SLC under identical injection conditions with the overall linac phase adjusted to minimize the final energy spread. Courtesy J. Seeman (2000).

Case iii: $W_{\perp}=W_{\perp}{ }^{\prime} z$ - wakefield linear along the bunch's longitudinal extent $E=E_{0}$ or $\frac{d E}{d s}=0$ - no acceleration $k=k_{0}$ - constant gradient

The equation of motion is

$$
x^{\prime \prime}(z, s)+k_{0}^{2} x(z, s)=\frac{e^{2}}{E(s)} \int_{z}^{\infty} \rho\left(z^{\prime}\right) W_{\perp}\left(z^{\prime}-z\right) x\left(z^{\prime}, s\right) d z^{\prime} .
$$

The essential features of the general solution [35] may be visualized using a simplified macroparticle model $[1,8]$. Here, the bunch is divided into three slices each having a rectangular distribution. The head $(h)$ has charge $\frac{N}{4}$, the core $(c)$ has 
charge $\frac{N}{2}$, and the tail $(t)$ charge $\frac{N}{4}$. The core is separated from the neighboring slices by the rms bunch length $\sigma_{z}$. The equations of motion for each macroparticle are

$$
\begin{aligned}
x_{h}^{\prime \prime}+k_{0}^{2} x_{h} & =0 \text { by causality the leading slice has no driving term } \\
x_{c}^{\prime \prime}+k_{0}{ }^{2} x_{c} & =B x_{h} \text { the driving term is given by the effect of the head } \\
& \text { on the core } \\
x_{t}^{\prime \prime}+k_{0}{ }^{2} x_{t} & =2 B x_{h}+2 B x_{c},
\end{aligned}
$$

where in the last equation the first term has a factor of 2 representing the $2 \sigma_{z}$ displacement of the head relative to the core, and the second term has a factor of 2 since the core has twice the charge of the head. The factor $B$ is

$$
B=\frac{e^{2}}{E}\left(\frac{N}{4}\right) W_{\perp} \sigma_{z}
$$

The solutions for Eq. 13 with initiappeal condition $x^{\prime}(s=0)=k_{0} \hat{x}$ are

$$
\begin{aligned}
& x_{h}=\hat{x} \sin k_{0} s \\
& x_{c}=\hat{x}\left[\left(1+\frac{B}{4 k_{0}^{2}}\right) \sin k_{0} s-\frac{B}{2 k_{0}} s \cos k_{0} s\right] \\
& x_{t}=\hat{x}\left[\left(1+\frac{2 B}{k_{0}^{2}}\right) \sin k_{0} s-\frac{2 B}{k_{0}} s \cos k_{0} s-\frac{B^{2}}{4{k_{0}}^{2}} s^{2} \sin k_{0} s\right] .
\end{aligned}
$$

In the 3-slice macroparticle model the amplitudes of the head, tail, and core are all linear in the initial displacement $\hat{x}$. Each slice adds an additional power of $(B s)$ which is proportional to the product $N W_{\perp} s$ which suggests an exponential growth of the tail of the beam in the limit of many slices [8]. Shown in Fig. 5 are profile monitor measurements and trajectories for three different initial vertical displacements (settings of a vertical dipole corrector magnet) from the SLC [8]. The middle plots correspond to an optimized orbit. In the top plots the beam is kicked in one direction and in the bottom plot in the other direction. The increase in vertical amplitude towards the tail of the bunch shows the intrabunch particle displacements due to the transverse wakefields as described in Eq. 15. Also evident from this measurement is a position-energy correlation ${ }^{2}$. The observed decrease in energy along the bunch depends subtely on the cancellation between the rf slope and the slope of the accelerating rf. This will be discussed further in the next section.

While the emittance of a slice of the beam in Fig. 5 seems nearly preserved, for experiments the projected emittance (seen by projecting the distribution onto the $y$ axis) is important and is observed here to be larger than the slice emittances.

2) these measurements were obtained by deflecting the beam onto a fluorescent screen using a kicker magnet located in a dispersive region (in the collider arcs) so that the measured horizontal position indicates an energy deviation; i.e. the profile monitor shows $y(E)$. 


\section{Increasing Energy \\ Bunch Tail Bunch Head}
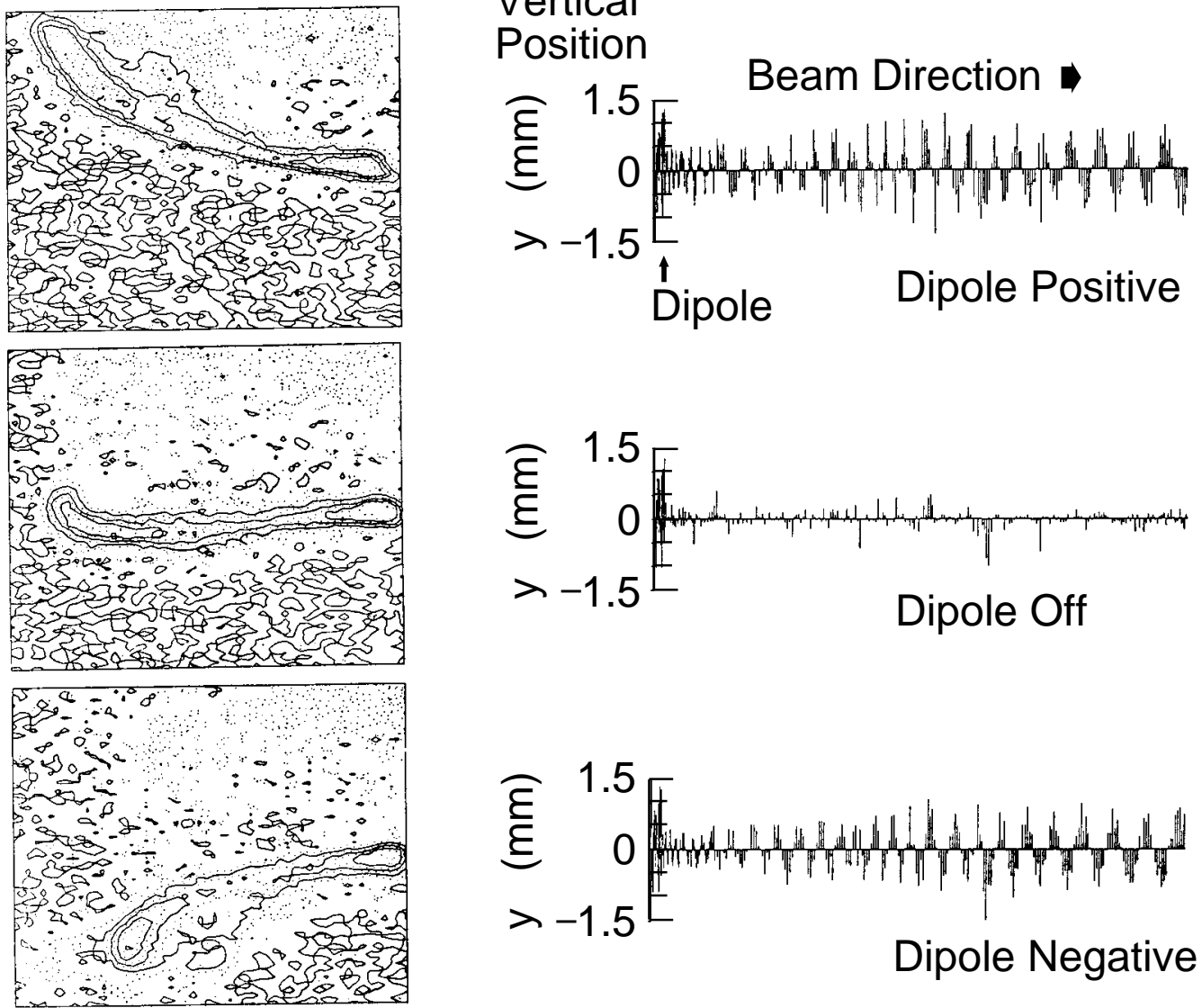

FIGURE 5. Profile monitor measurements in a region of nonzero dispersion after the end of the SLC linac and vertical centroid trajectories with a positive perturbation to the particle orbit (top), under nominal conditions (middle), and with a negative perturbation (bottom). Courtesy J. Seeman (2000).

Shown in Fig. 6 are now the transverse beam profiles measured at the end of the SLC linac for various initial beam displacements [36]. These measurements were made [37] by deflecting the beam using fast kicker magnets located within the linac so that the true transverse profile $y(x)$ is represented. Based on the above analysis we may interpret the faint tail seen with large amplitude excitations as the off-energy and off-axis tail generated by the transverse wakefields.

Case iv: $W_{\perp}=W_{\perp}^{\prime} z=W_{\perp} z / l$

$$
\frac{d E}{d s} \neq 0 \text { or } E=E_{0}(1+G s)
$$



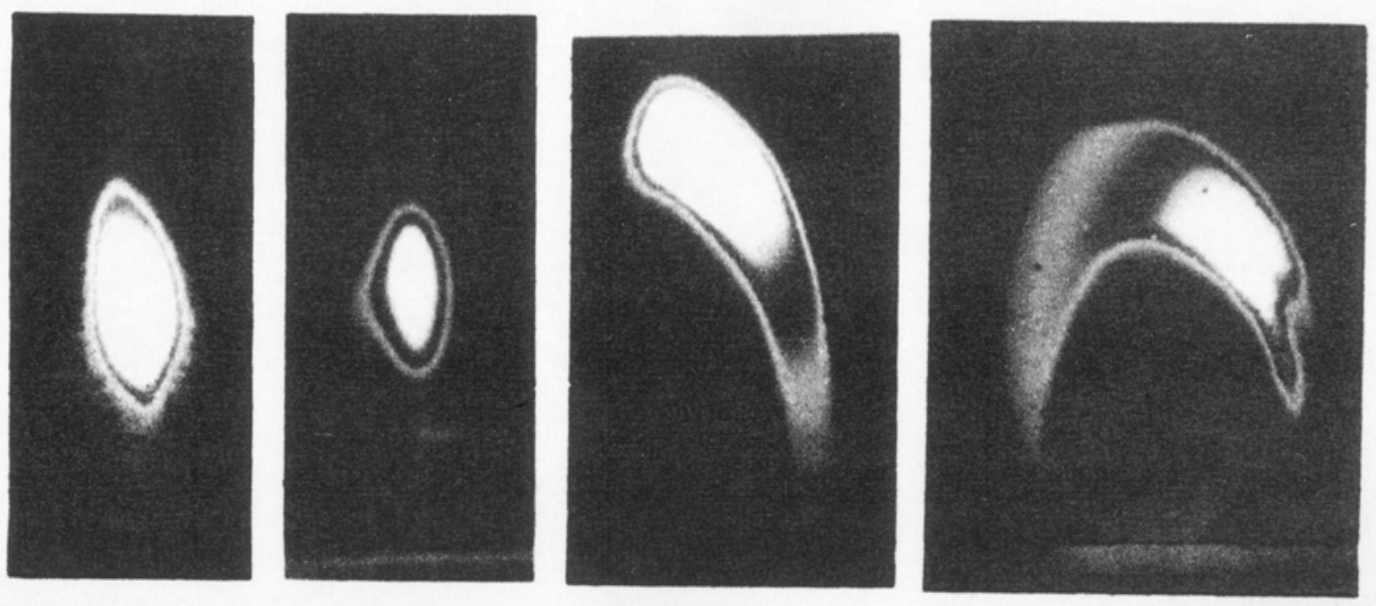

$9-89$

$6438 A 6$

FIGURE 6. Measured beam profiles demonstrating emittance growth due to wakefields as a function of increasing oscillation amplitude. From left to right the amplitudes in the applied horizontal trajectory displacement are $0 \mathrm{~mm}, 0.2 \mathrm{~mm}, 0.5 \mathrm{~mm}$, and $1.0 \mathrm{~mm}$. The single-bunch charge was $2 \times 10^{10}$ electrons. Courtesy J. Seeman (2000).

\section{(a) $k$ adiabatic ${ }^{3}$}

The solution to the general equation of motion has no closed form expression. It is obtained [35] by expanding the solution $x(z, s)$ in a power series and solving recursively. In the asymptotic limit of strong wakefields $(|\eta|>>1)$, the peak-toinitial amplitude given at the end of the linac of length $L$ is [38]

$$
\frac{x(z, L)}{x_{0}}=\sqrt{\frac{E_{0} k_{0}}{E(s) k(s)}} \frac{\eta^{-\frac{1}{6}}}{\sqrt{6 \pi}} e^{\frac{3 \sqrt{3}}{4}} \eta^{\frac{1}{3}},
$$

where

$$
\eta(z)=\frac{e N W_{\perp}^{\prime} z^{2}}{\sigma_{z}} \int_{0}^{L} \frac{d s}{E(s) k(s)}>>1 .
$$

The last 2 equations show the lagging particle trajectory increasing exponentially with the transverse wakefield. Based on such observations first made at the SLC [39]- [41], this phenomenon has come to be referred to as beam breakup [39]- [42].

Another example of large transverse beam tails is shown [8] in Fig. 7. In this measurement the bunch was intentionally lengthened to about $2.5 \sigma_{z}$ or $2.5 \mathrm{~mm}$ and made to oscillate both horizontally and vertically to sample the transverse wakefields. The profile shows $y(x)$. The head of the beam is at the lower right while the tail of the beam contributes significantly to larger projected beam emittances 


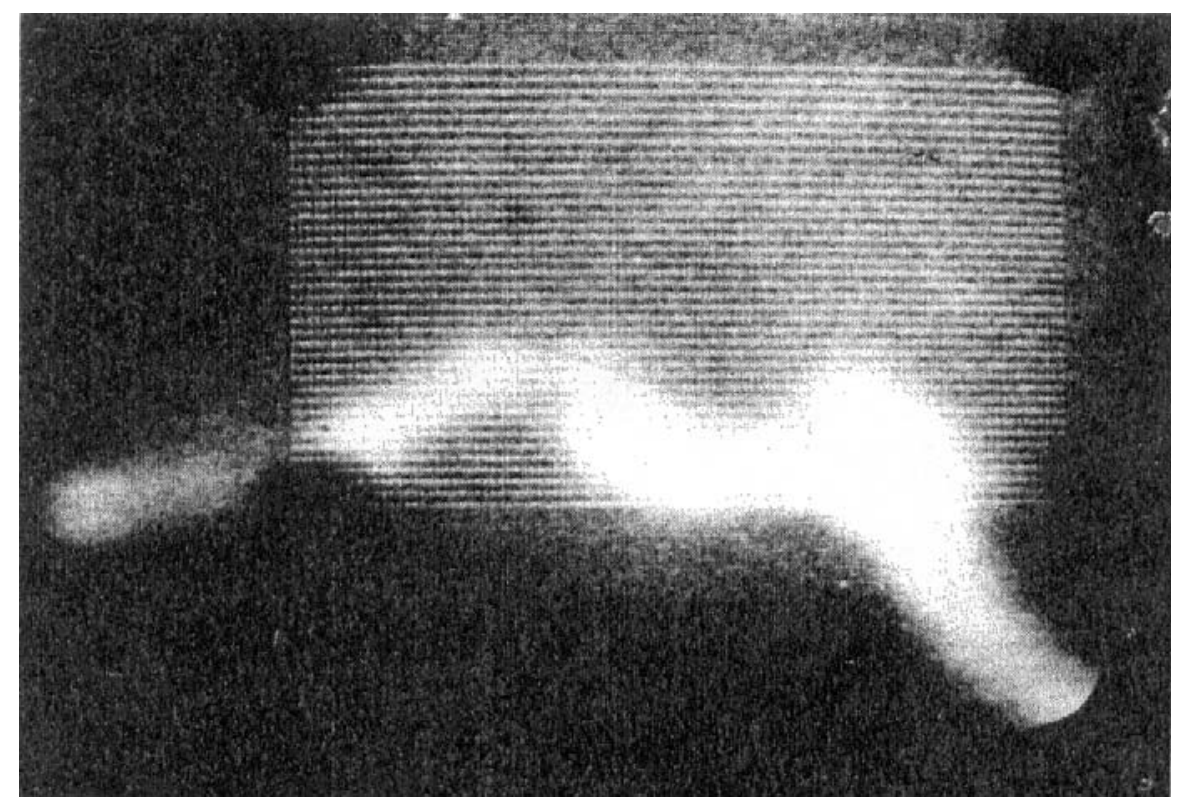

FIGURE 7. Measured transverse profile of a long bunch showing large transerverse displacements of the bunch tail. Courtesy J. Seeman (2000).

in both transverse planes.

\section{(b) $k$ tailored}

The cure for the beam breakup instability may be motivated as the follows. With the same focussing function $k$ experienced by all the particles within a bunch, a perturbation at the head of the bunch due to the transverse wakefield may resonantly drive particles in the tail of the bunch. By making the focussing function different across the bunch, such resonant build-up can be avoided. This is precisely the technique proposed by Balakin, Novokhatsky, and Smirnov known today as $B N S$ damping [43] in their honor. Using the 3-slice model of ref. [1,8], the head of the bunch is focussed with $k=k_{0}+\alpha$, the core with $k=k_{0}$, and the tail with $k=k_{0}-\eta$. Requiring $[1,43]$ that the head and core follow the same trajectories, the solutions for $\alpha$ and $\eta$ are

$$
\alpha=e \frac{N}{4} \frac{W_{\perp} \sigma_{z}}{E_{0} k_{0}}, \text { core follows head, and } \eta=4 \alpha .
$$

At the SLC the variation in $k$ was achieved by back-phasing the first part of the linac (i.e. the bunch preceeds in time the rf wave) and, to restore a small energy spread at the linac end, by forward phasing the remainder of the linac. This introduced a

3) $k$ is taken to scale with energy such that the instantaneous wavelength $\lambda(s)=2 \pi / k$ is constant; i.e. $\lambda(s)=\lambda_{0}$. 
correlated energy spread within the bunch as shown [44] in Fig. 8 which is just as desired to achieve BNS damping on a global scale. The optimum choice of phases is current-dependent and was determined by measuring the peak amplitude response at the end of the linac following a perturbation applied after injection.

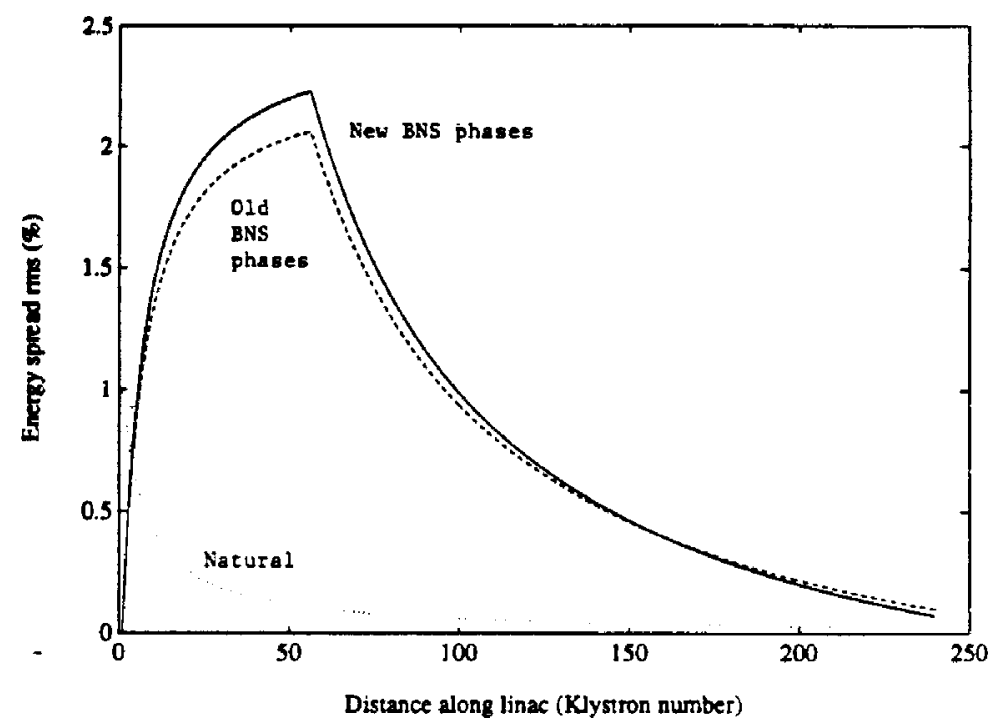

FIGURE 8. Estimated energy spread at the end of the linac (i.e. a projection along the horizontal axis of Fig. 5) for different complements of linac klystron rf phases. The two different curves are optimized for two different bunch currents. Courtesy F.J. Decker (2000).

Measured transverse centroid oscillations both with and without BNS damping invoked are shown [1] in Fig. 9 following an intentionally applied initial displacement early in the SLC linac. The measured centroid displacement normalized to initial kick amplitude was reduced by about a factor of 10 with BNS damping implemented.

(c) $k$ exact

With BNS damping alone, the projected 6-dimensional beam emittance may assume large values along the linac where the beam energy spread is large. Subsequent emittance dilution may also occur if the dispersion is not perfectly corrected. Alternatively, a general condition on the focussing $k$ may be determined. Substituting $x(s)=\hat{x_{0}} \cos \left(k_{0} s+\phi_{0}\right)$ in Eq. 2, leads to $[43,45]$

$$
k_{0}^{2}=k^{2}(z, s)+\frac{r_{0}}{\gamma(z, s)} \int_{z}^{\infty} \rho\left(z^{\prime}\right) W_{\perp}\left(z^{\prime}-z\right) x\left(z^{\prime}, s\right) d z^{\prime} .
$$

If $k(z, s)$ can be adjusted to exactly compensate the second term in Eq. 19 for all particles within the bunch, then the particles within the bunch follow the same trajectory and experience the same focusing. This allows the small projected 6dimensional beam emittance to be maintained over the entire length of the linac.

The condition given in Eq. 19 is refered to as auto-phasing and is hard to realize in practice [8]. The adjustable parameters are the lattice focussing $k$, the beam 


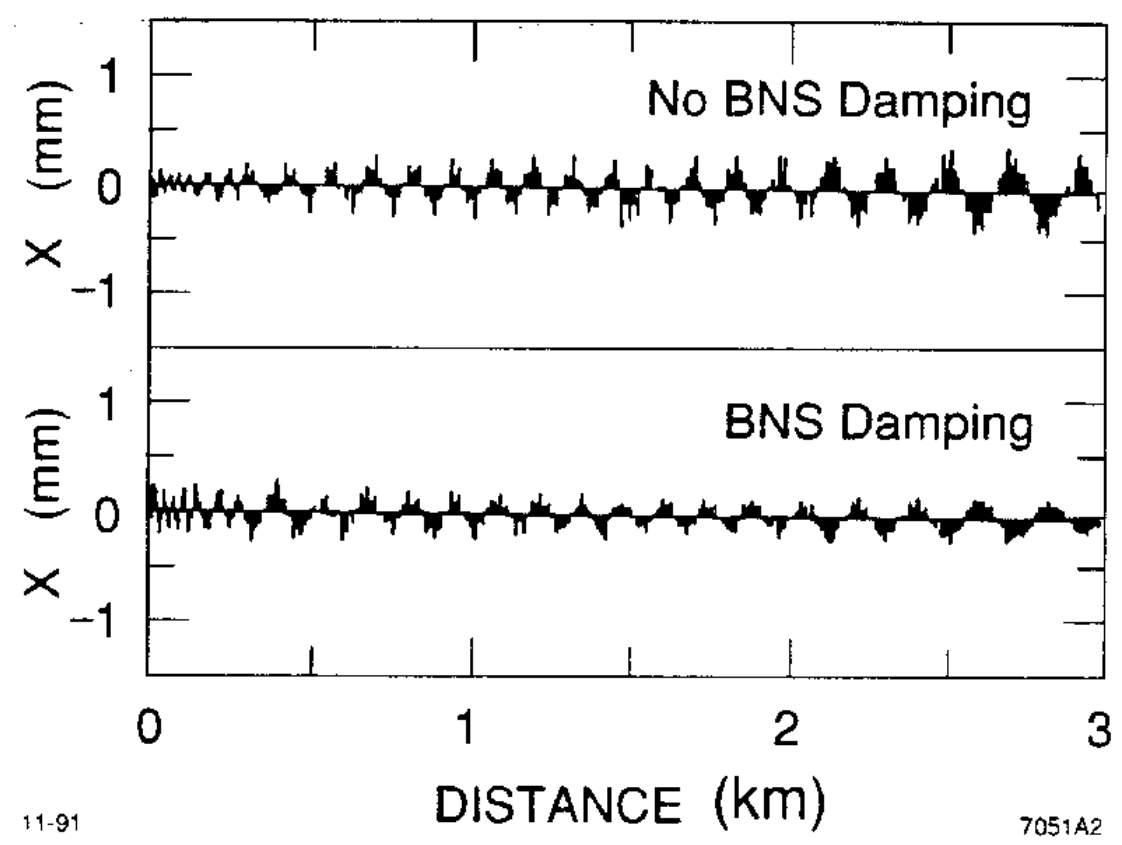

FIGURE 9. Measured horizontal trajectories obtained under nominally identical conditions without BNS damping (top) and with BNS damping (bottom) at $2 \times 10^{10}$ particles. Courtesy J. Seeman (2000).

energy $\gamma$, and the longitudinal profile $\rho$. Of these, the easiest to control is the longitudinal profile, which is the main topic of the next section.

\section{LONGITUDINAL EMITTANCE PRESERVATION}

The energy of the particles with longitudinal density distribution $\rho(z)$ is a sum of the injected beam energy $E_{0}$, the energy gained in acceleration from each klystron $\Delta E_{i}$, and the losses from longitudinal wakefields $W_{\|}[31,45]$ :

$$
E(z)=E_{0}+\sum_{i=1}^{N_{k l y s}}\left[\Delta E_{i} \cos \left(\phi_{i}+\phi(z)\right)+\Delta s_{i} \int_{z}^{\infty} W^{i}{ }_{\|}\left(z^{\prime}-z\right) \rho\left(z^{\prime}\right) d z^{\prime}\right],
$$

where $\phi_{i}$ is the klystron phase (i.e. the arrival time of the beam with respect to the crest of the rf), $\phi(z)=\frac{2 \pi z}{\lambda_{r f}}, \lambda_{r f}=\frac{c}{f_{r f}}$ is the rf wavelength at frequency $f_{r f}$, and $\Delta s$ gives the distance between klystrons.

The energy spread of the bunch $\sigma_{E}$ is obtained by averaging over the particle distribution after subtracting out the mean energy $<E>$ of the bunch. Normalized to the mean energy

$$
\frac{\sigma_{E}}{E}=\frac{1}{<E>}\left[\int_{-\infty}^{\infty}(E(z)-<E>)^{2} \rho(z) d z\right]^{\frac{1}{2}}
$$


where the mean energy of the bunch is

$$
<E>=\int_{-\infty}^{\infty} E(z) \rho(z) d z .
$$

In the low-current limit $\left(\rho(x) \sim 0, W_{\perp}=0, W_{\|}=0\right)$, the beam does not take away any energy from the accelerating structures and the beam is placed on the crest of the rf wave to achieve both maximum acceleration and minimum energy spread within the bunch. At higher beam currents while invoking BNS damping $\left(\rho(x) \neq 0, W_{\perp} \neq 0\right)$ with $W_{\|}=0$, the klystrons in the first part of the linac are phased to impart relatively higher energy to the head of the bunch while in the later part of the linac the energy spread is restored (recall Fig. 8).

At even higher bunch currents, $\left(\rho(x) \neq 0, W_{\perp} \neq 0\right)$ with $W_{\|} \neq 0$ one must carefully balance the second and third terms in Eq. 20; that is cancel the energy variation along the bunch arising from the slope of the rf and that from the longitudinal wakefield. Shown in Fig. 10 are sketches illustrating such cancellation. The effective rf gain representing the vector sum of all accelerating stations is plotted versus time together with the projection of the charge distribution which shows the resultant energy spread of the bunch. At low current, a bunch placed on crest has minimum energy spread. Off crest, there is a position-energy correlation and the energy spread is increased. At high current, due to longitudinal wakefield, or beam loading, a bunch placed on crest has a large energy spread. For short, high intensity bunches, the energy spread may be minimized by placing the beam off-crest as shown. In this case the beam-induced wakefield exactly cancels the slope of the rf across the bunch.
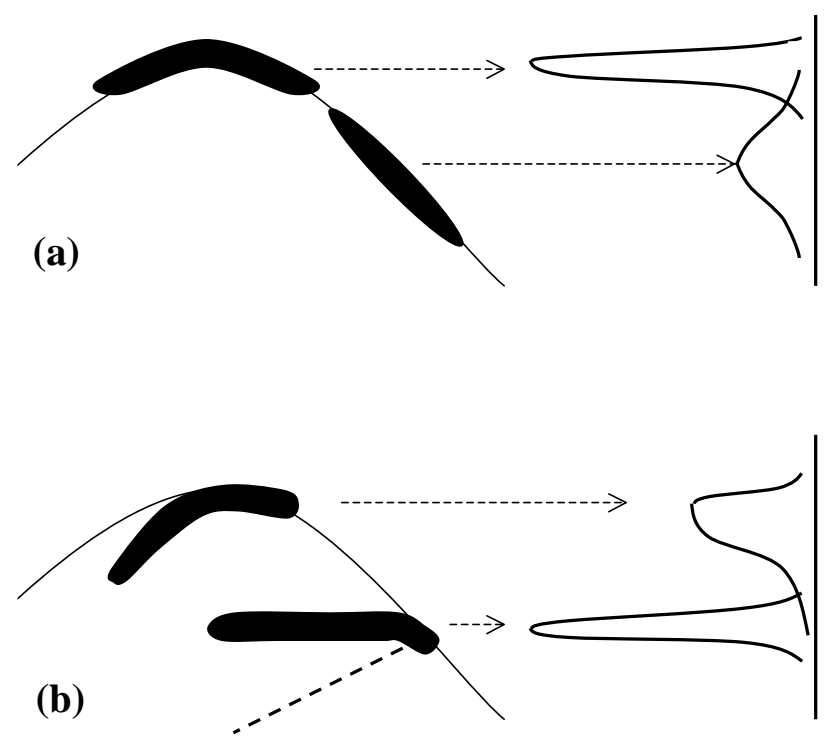

FIGURE 10. Effective energy gain and energy spread for low (a) and high (b) current bunches illustrating optimum klystron phasing for minimum energy spread. 
Measurements of the beam energy spread at the end of the linac versus klystron phase may be used to determine the optimum phase settings. An example [46] is given in Fig. 11. The case of highest energy gain, case (f), corresponds to Fig. 10b for the on-crest bunch. As the rf phase was varied an optimum condition, case (b), was attained albeit with a long low-energy tail.

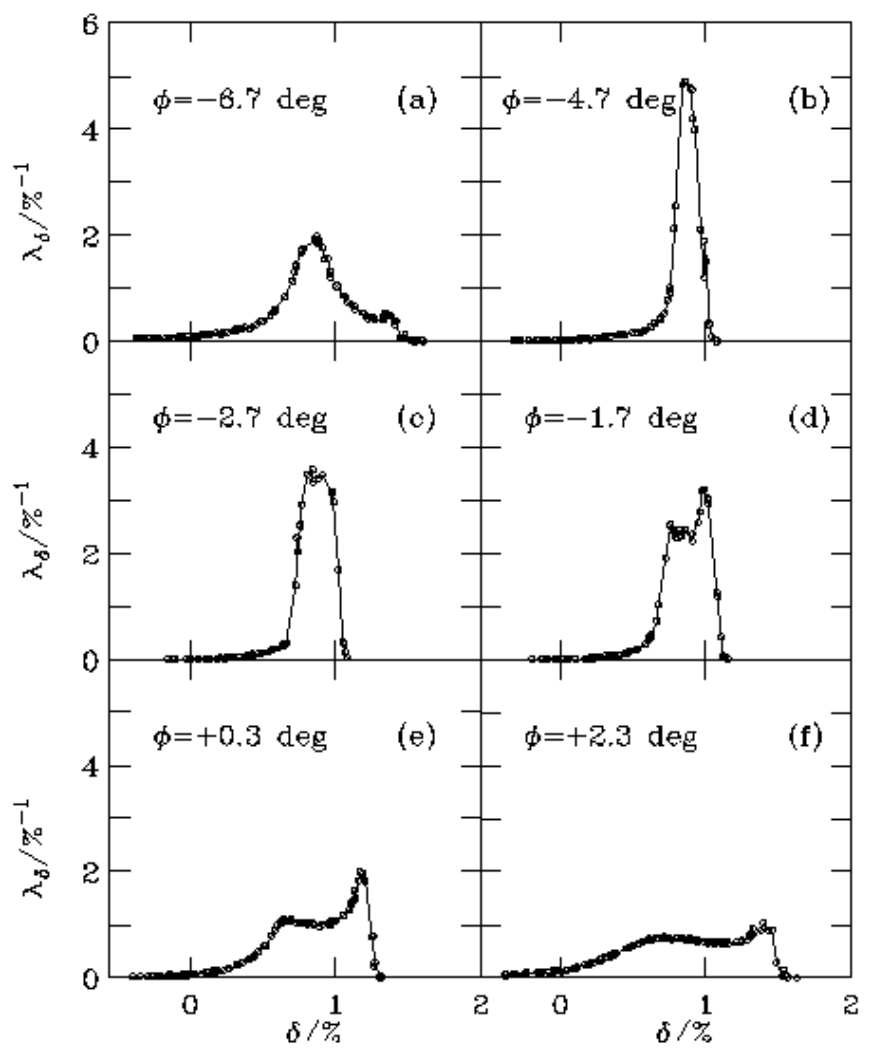

FIGURE 11. Measured energy spread at the end of the SLC linac for various relative overall linac phase. The vertical axis shows relative intensity of the energy distribution of the beam while the horizontal axis shows the relative beam energy spread. Maximum accelerating gradient, or zero absolute phase with respect to the rf crest, is seen in subplot (f). Courtesy K. Bane (2000).

Depending on the bunch length and charge the cancellation may be imperfect resulting in a nonlinear energy variation across the bunch which, as can be seen in Fig. 11, results in non-Gaussian energy distributions with energy 'tails'. Such energy tails have proven to be highly detrimental to collider performance. Highly motivated to minimize chromatic effects in the downstream final focus systems, commensurate measures are always taken to understand the longitudinal beam dynamics [47-50] and to avoid such tails in the beams' energy distribution. This may be achieved by shortening the bunch using bunch compression, by shaping the bunch distribution to modify the wakefield-driven term in Eq. 20 using bunch shaping or possibly using a combination in a scheme refered to as over compression. 


\section{Bunch Compression}

To minimize the effects of imperfect cancellation between the accelerating $\mathrm{rf}$ and the longitudinal wakefields, the bunch length is made shorter before injection into the main linac using bunch compressors. A schematic view of a bunch compressor is shown in Fig. 12. It consists of a transport line with initially zero dispersion containing an acceleration structure. The bunch first passes the accelerating cavity at the zero crossing (that is, there is no net energy gain averaged over the bunch) which introduces an energy-position correlation along the bunch. The bunch is then transported through bending dipoles. The inherent energy-dependent path length of the transport line is such that particles at the front of the bunch travel a longer distance while particles in the tail travel a shorter distance which produces a compression of the bunch length.

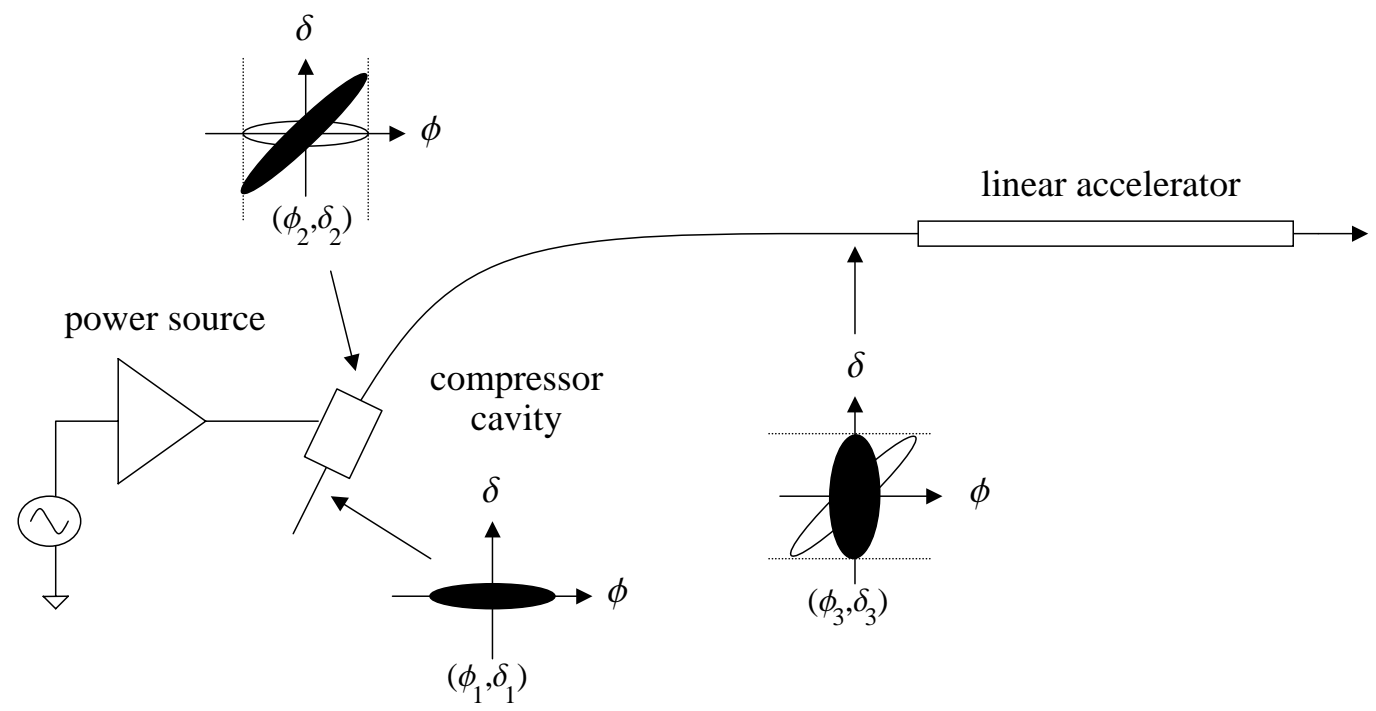

FIGURE 12. Schematic illustration of the bunch compression scheme implemented at the SLC (see also Ref. [49]).

For illustration, the bunch length at the entrance to the linac is evaluated. Initially, the bunch enters the 'compressor cavity' with particle coordinates $\left(\phi_{1}=\right.$ $\left.\frac{\omega z}{c}=\frac{\omega}{c}\left(\frac{\lambda_{1}}{2 \pi}\right), \delta_{1}\right)$. At the exit of the cavity,

$$
\begin{aligned}
\phi_{2} & =\phi_{1} \\
\delta_{2} & =\delta_{1}+\frac{e V}{E} \sin \phi_{1}
\end{aligned}
$$

At the entrance to the linac, after passing through the dispersive region,

$$
\begin{aligned}
\phi_{3} & =\phi_{2}-\frac{R_{56} \omega}{c} \delta_{2} \\
\delta_{3} & =\delta_{2},
\end{aligned}
$$


where $R_{56}=d z / d \delta$ represents the lattice property which translates energy into longitudinal displacement. Combining Eqs. 23-24,

$$
\phi_{3}=\phi_{1}-R_{56}\left(\delta_{1}+\frac{e V}{E} \sin \phi_{1}\right) \approx \phi_{1}\left(1-\frac{R_{56} e V \omega}{E c}\right)-R_{56} \delta_{1} .
$$

The bunch length at the entrance to the linac is

$$
\begin{aligned}
\sigma_{z_{3}} & =\frac{\omega}{c} \sigma_{\phi_{3}} \equiv \frac{\omega}{c}<\phi_{3}^{2}>^{\frac{1}{2}} \\
& =\frac{\omega}{c}\left[\left(1-R_{56} \frac{e V \omega}{E c}\right)^{2}<\phi_{1}^{2}>-2 R_{56}\left(1-\frac{R_{56} e V \omega}{E c}\right)<\phi_{1} \delta_{1}>+R_{56}{ }^{2}<\delta_{1}{ }^{2}>\right]^{\frac{1}{2}} \\
& \approx \sqrt{\left(1-\frac{\omega}{c} R_{56} \frac{e V}{E}\right)^{2} \sigma_{z 1}^{2}+R_{56}^{2} \sigma^{2}{ }_{\delta 1}} \approx\left(1-\frac{\omega}{c} R_{56} \frac{e V}{E}\right) \sigma_{z_{1}}
\end{aligned}
$$

assuming $<\phi_{1} \delta_{1}>=0$ (i.e. there is no incoming $E-z$ correlation) and that the incoming energy spread is small so that $\left\langle\delta_{1}{ }^{2}>\right.$ is negligible. The trade-off between small bunch length and larger energy spread may be evaluated from $\sigma_{\delta}=<\delta_{3}{ }^{2}>^{\frac{1}{2}}$, which is usually not of importance. The above analysis holds provided that the incoming bunch length is small $\left(\sin \phi_{1} \sim \phi_{1}\right)$ so that only the linear portion of the accelerating voltage of the compressor cavity is seen by the beam.

\section{Bunch Shaping [51]}

The delicate process of canceling the effects of the slope of the rf and the energy gradient caused by beam loading may be simplified, in principle, by adjustments to the longitudinal charge distribution $\rho(z)$ in Eq. 20. The energy gained of a particle after traversing an accelerating structure [51] is

$$
E\left(\theta_{1}\right)=E_{0} \cos \theta_{1}+\int_{0}^{\left(\theta_{0}-\theta_{1}\right)} \rho\left(\theta^{\prime}\right) W_{\|}\left(\theta_{0}-\theta_{1}-\theta^{\prime}\right) d \theta^{\prime}
$$

where $\theta_{0}$ is the position of the head of the bunch and $\theta^{\prime}$ varies from 0 at the head of the bunch to $\theta_{0}-\theta_{1}$ where the net energy is evaluated. To reduce the energy spread within the bunch, $e V\left(\theta_{1}\right)=E\left(\theta_{1}\right)$ should be independent of $\theta_{1}$;

$$
\frac{\partial V\left(\theta_{1}\right)}{\partial \theta_{1}}=0
$$

Using a realistic model of the longitudinal wakefield of the SLC structures, this equation has been solved for the charge distribution as shown [51] in Fig 13. The horizontal axis shows the phase of individual particles with respect to the head of the bunch (located at phase zero) while the different curves show different phase offsets of the bunch head relative to the crest of the rf. The points marked by ' $\mathrm{T}$ ' indicate the extent of the bunch for a total of $5 \times 10^{10}$ particles. For short bunches with large phase offsets (as needed for BNS damping), Fig. 13 shows that a charge distribution peaked towards the head of the bunch is desirable. 


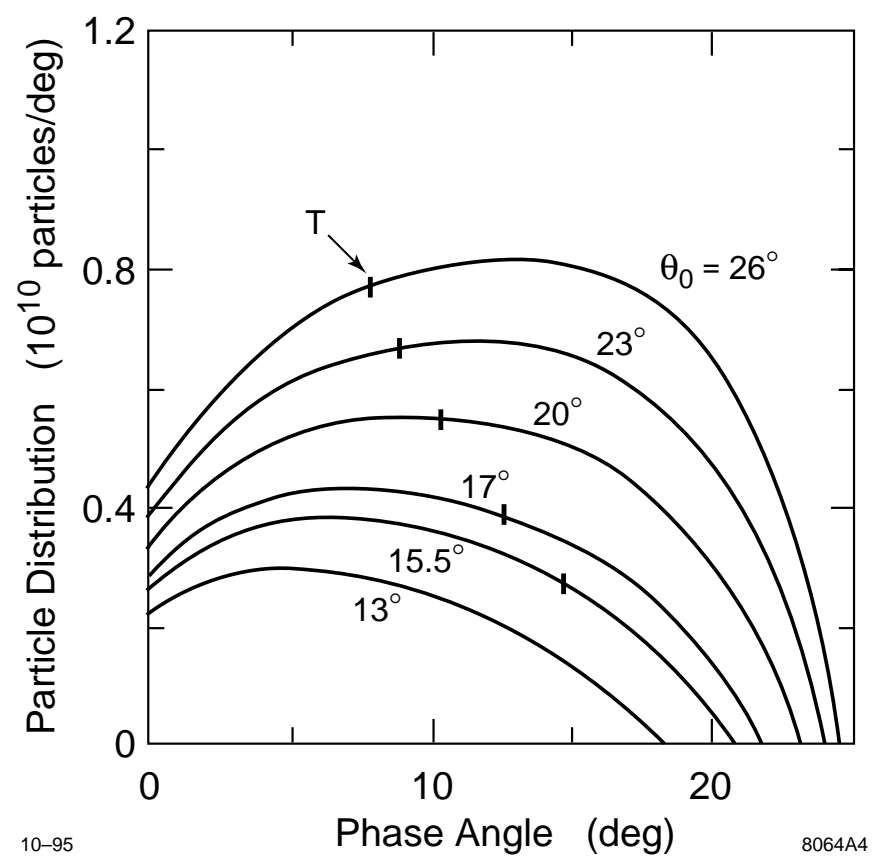

FIGURE 13. Particle distribution, or bunch shape, as a function of phase angle for minimum energy spread. Courtesy G. Loew (2000).

\section{Overcompression}

Overcompression [52,48-50] using the bunch compressor was used at the SLC for minimization of the energy tails using a combination of bunch compression and bunch shaping. It served to be particularly useful in the case that $\sin \phi_{1} \neq$ $\phi_{1}$ in Eq. 25; that is for the case that the nonlinear fields of the compressor cavity are important. It is hoped that future linear collider designs make note of the unfortunate consequences of bunch lengthening in the upstream damping ring systems (see [24]- [29]) and avoid having to treat such nonlinear effects.

Shown [52] in Fig. 14 are simulations of the longitudinal phase space and resultant energy spread at the entrance to the linac taking into account the nonlinear rf fields seen by a long bunch of length $1 / 10$ that of the compressor cavity rf wavelength. The case of undercompression is shown on the left while the distributions for overcompression at higher compressor cavity voltage on the right. Overcompression yields two advantages: reduced particles in the energy tails as well as a steeper rise in the longitudinal distribution at the head of the bunch. The corresponding rf waveform including beam loading and the energy spread calculated at the end of the linac are shown [52] in Fig. 15. On the left, the under-compressed bunch has a longitudinal distribution which does not cancel the slope of the rf. The result is an extended low energy tail in the energy spectrum. With overcompression, the slope of the rf and the beam loading compensate one another nearly perfectly resulting in a small energy spread at the end of the linac. 

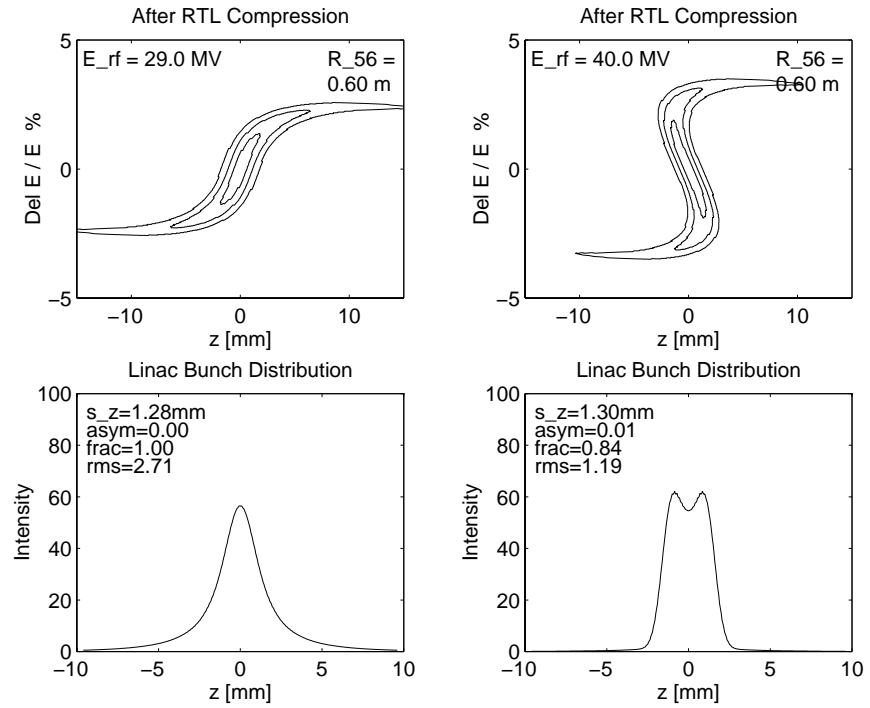

FIGURE 14. Simulation of the longitudinal phase space and beam energy distribution at injection into the linac with bunch undercompression (left) at $85 \%$ of nominal compression voltage and bunch overcompression (right) at $120 \%$ of nominal voltage. The injected bunch length was taken to be $10 \mathrm{~mm}$, or approximately 1/10th of the rf wavelength. Courtesy F.J. Decker (2000).
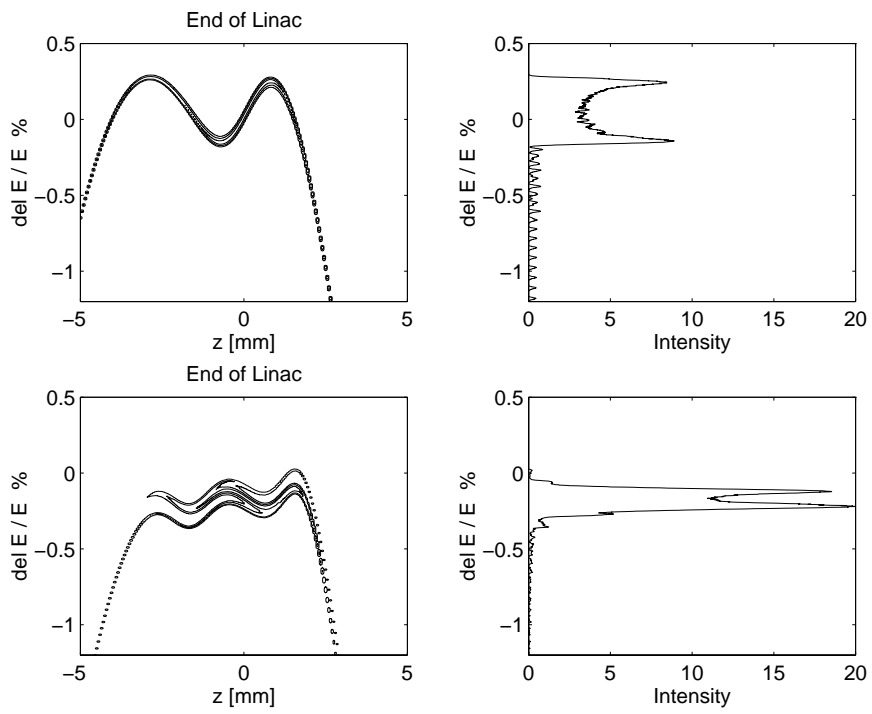

FIGURE 15. Simulation of the rf waveform and beam energy distributions at the end of the linac with bunch undercompression (top) and bunch overcompression(bottom). Courtesy F.J. Decker (2000). 
Measurements of the beam at the end of the linac in a dispersive region are shown [52] in Fig. 16 with undercompression (left) and with overcompression (right). The absence of the low energy tails justified the routine use of over-compressed beams at the SLC.
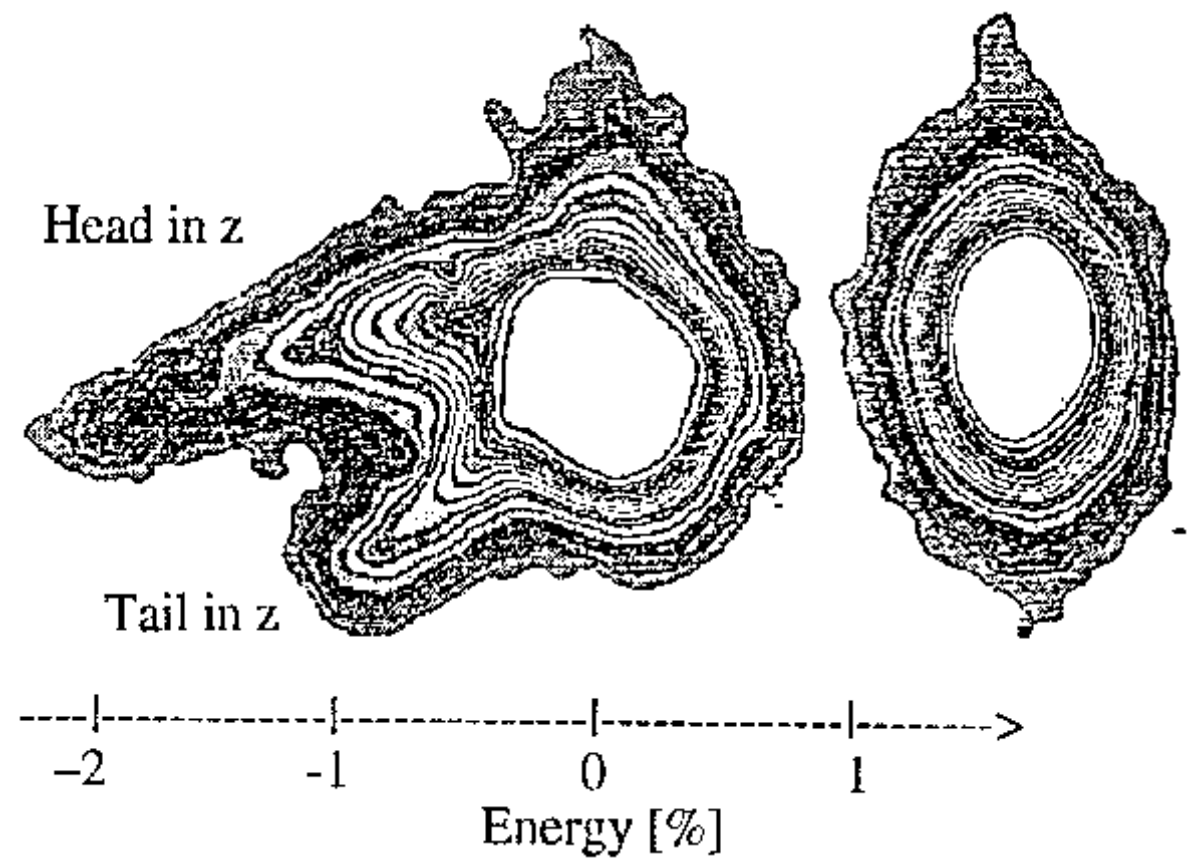

FIGURE 16. Measurements of the beam profile at a dispersive location at low compressor voltage (left) and with bunch overcompression (right). Courtesy F.J. Decker (2000).

\section{TRAJECTORY STEERING}

Motivation and perspective for this section is given with the following considerations. Recall from Appendix A that

$$
\epsilon=\frac{\sigma_{x}^{2}}{\beta} \text { with } \sigma_{x}^{2}=<x^{2}>^{\frac{1}{2}}
$$

As discussed previously, the horizontal $(x)$ or vertical $(y)$ trajectory is given by a superposition of terms (c.f. Eq. 4):

$$
\begin{aligned}
x & =x_{c}+x_{\beta}+x_{\eta} \\
& =x_{c}+x_{\beta}+\eta \delta .
\end{aligned}
$$

In chapter 2 was described how to minimize contributions from the term $x_{\beta}$ while chapter 3 focussed on minimization ${ }^{4}$ of $x_{\eta}$ by minimization of the beam energy

4) in the absence of cross-correlations of the form $\left\langle x_{c} x_{\eta}\right\rangle=0$, for example, the dispersive term $x_{\eta}=\eta \delta$ contributes in quadrature 
spread $\delta$. In this section steering procedures which aim to minimize emittance dilutions arising from deviations in the central trajectory $x_{c}$ and from the dispersion $\eta$ will be described.

Increased beam emittances may arise from beam-to-magnet or beam-to-structure position deviations as shown conceptually in Fig. 17. The beam passing through a single misplaced quadrupole experiences the next lower-order field namely a dipole field $\left(<x_{c} x_{\beta}>\neq 0\right)$. The misalignment therefore generates a betatron oscillation and dispersion as higher energy particles are less deflected by the dipole field. In the case of a displaced structure a betatron oscillation is also induced due to the transverse wakefield. Recall that the driving term is linear in the wakefield times the initial displacement. In either case, the ensuing orbit is such that further emittance dilutions may result downstream of the perturbation due to the initial errors.

(a)

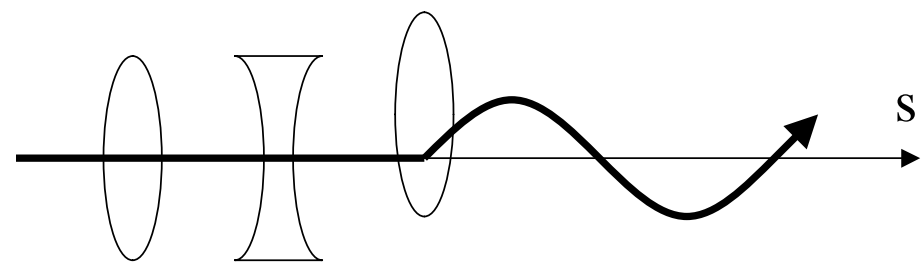

(b)

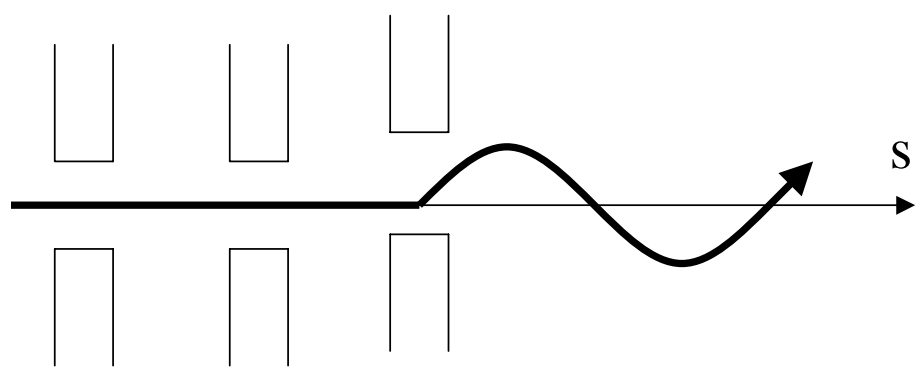

FIGURE 17. Conceptual drawing illustrating orbit perturbations due to misaligned quadrupoles (a) or structures (b).

\section{A One-to-one steering}

This algorithm aims to steer the beam so that the transverse displacements measured by beam position monitors (BPMs) are minimized. The BPMs are typically mounted near the center of quadrupoles since their sensitivity is highest at large $\beta$-function. A conceptual orbit steered one-to-one is shown in Fig. 18. The beam is successfully kicked to pass through the magnet center and, assuming that the

$$
\sigma_{\eta}=\left\langle x_{\eta}{ }^{2}>^{\frac{1}{2}}=\eta^{2}<\delta^{2}>^{\frac{1}{2}} .\right.
$$


BPM is not offset with respect to the quadrupole, the BPM would show zero displacement. Notice however that one-to-one steering generates dispersion which contributes to emittance dilutions.

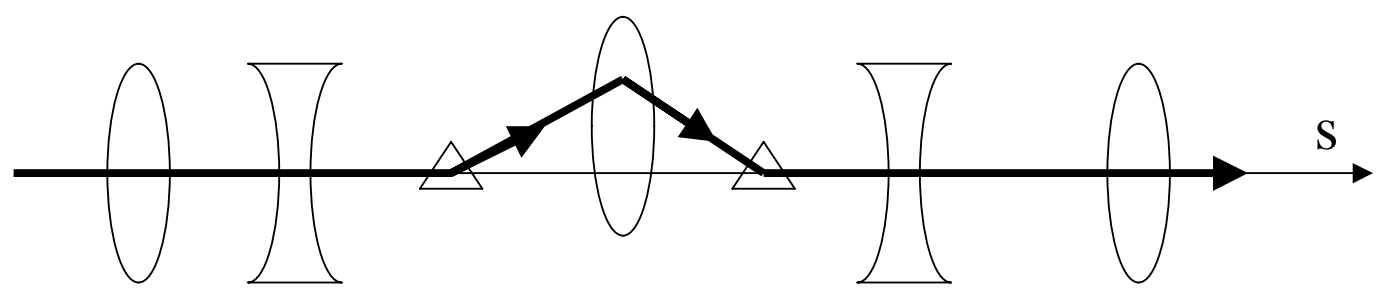

FIGURE 18. Conceptual illustration of a closed bump that would minimize the BPM reading after one-to-one steering.

In a transport line the beam centroid position measured downstream at location $s=j$ obeys

$$
x_{j}=\sum_{i=0}^{j} \sqrt{\beta_{i} \beta_{j}} \theta_{i} \sin \left(\theta_{j}-\theta_{i}\right),
$$

which has contributions from each dipole kick $\theta_{i}$ and depends on the $\beta$-functions at the location of the initial disturbance $(i)$ and at the observation point $(j)$. The corrector magnet fields to be applied to minimize the BPM readings will be solved for assuming linear transport; that is, that there are no nonlinear magnetic fields and the measurements are made at low bunch current so that nonlinear wakefield effects may be ignored.

In matrix form

$$
\vec{x}=M \vec{\theta},
$$

where $\vec{x}$ is the set of measurements from $m$ BPMs, $\vec{\theta}$ is the set of kick angles to be applied by $n$ correctors, and $M$ contains the transfer matrix elements between the correctors and the BPMs:

$$
\begin{aligned}
x^{T} & =\left(x_{0}, x_{1}, \ldots, x_{m}\right) \\
\theta^{T} & =\left(\theta_{0}, \theta_{1}, \ldots, \theta_{n}\right) \\
M_{i j} & =\sqrt{\beta_{i} \beta_{j}} \sin \left(\phi_{j}-\phi_{i}\right)
\end{aligned}
$$

Solving Eq. 37 the kick angles to be applied for minimizing the BPM readings are obtained:

$$
M^{T} \vec{x}=M^{T} M \vec{\theta} \text { or } \vec{\theta}=\left(M^{T} M\right)^{-1} M^{T} \vec{x} .
$$

If the number of correctors equals the number of BPMs then $M$ is a square matrix so Eq. 37 reduces to simply $\vec{\theta}=M^{-1} \vec{x}$. Otherwise the general form is taken. If 
$n>m$ the matrix is overdetermined. For $n<m$ the number of unknowns exceeds the number of measurements so an independent measurement should be made after changing some parameter, for example, the beam energy. In a linear accelerator Eq. 32 must be modified [53] to include the energy scaling factor $\sqrt{\frac{E_{i}}{E_{j}}}$. This introduces $m$ additional unknowns so additional measurements are required to constrain the solution.

As motivation for the algorithms to be used below in the discussion of beambased alignment and dispersion-free steering, the solution is equivalently formulated in terms of a minimization procedure, which is well adapted to computational evaluation. The function to be minimized, given by Eq. 33, is

$$
\sum_{j}\left[x_{j}-\sum_{i} M_{i j} \theta_{i}\right]^{2}
$$

where $x_{j}$ again represents the BPM measurements and the fitting function $\sum_{i} M_{i j} \theta_{i}$ has unknowns $\theta_{i}$. The minimization procedure demands

$$
\begin{aligned}
0 & =\frac{\partial}{\partial \theta}\left[\sum_{j}\left(x_{j}-\sum_{i} M_{i j} \theta_{i}\right)^{2}\right] \\
& =2 \sum_{j}\left[x_{j}-\sum_{i} M_{i j} \theta_{i}\right] M_{k j}, \text { or } \\
\sum_{j} M_{k j} x_{j} & =\sum_{j} \sum_{i} M_{i j} M_{k j} \theta_{i},
\end{aligned}
$$

which is identical to Eq. 37.

\section{B Beam-based alignment}

We define beam-based steering algorithms as ones which provide information on magnet, BPM, or structure misalignments using measurements with the beam. With this definition, one-to-one steering may also be considered a beam-based alignment algorithm since the applied kicks $\theta$ are related to the quadrupole displacements $\Delta x$ by $\theta=k \Delta x$, where $k$ is the quadrupole focussing field.

More generally, we take in this example into account that the electrical zero of the BPMs may not be coincident with the magnetic center of the quadrupoles and that the quadrupoles themselves may be displaced with respect to the reference axis. The coordinate system used is sketched in Fig. 19. Here the beam position $x$ measured with respect to some reference axis, which is common to all magnets, is given as a sum of the quadrupole displacement $x_{q}$, the difference in location of the electrical center of the BPM and the magnetic center of the quadrupole $x_{b p m}$, and the measured BPM value $x_{m}$.

The beam position $x_{k}$ and angle $x_{k}{ }^{\prime}$ at quadrupole $k$ are given [7] by 


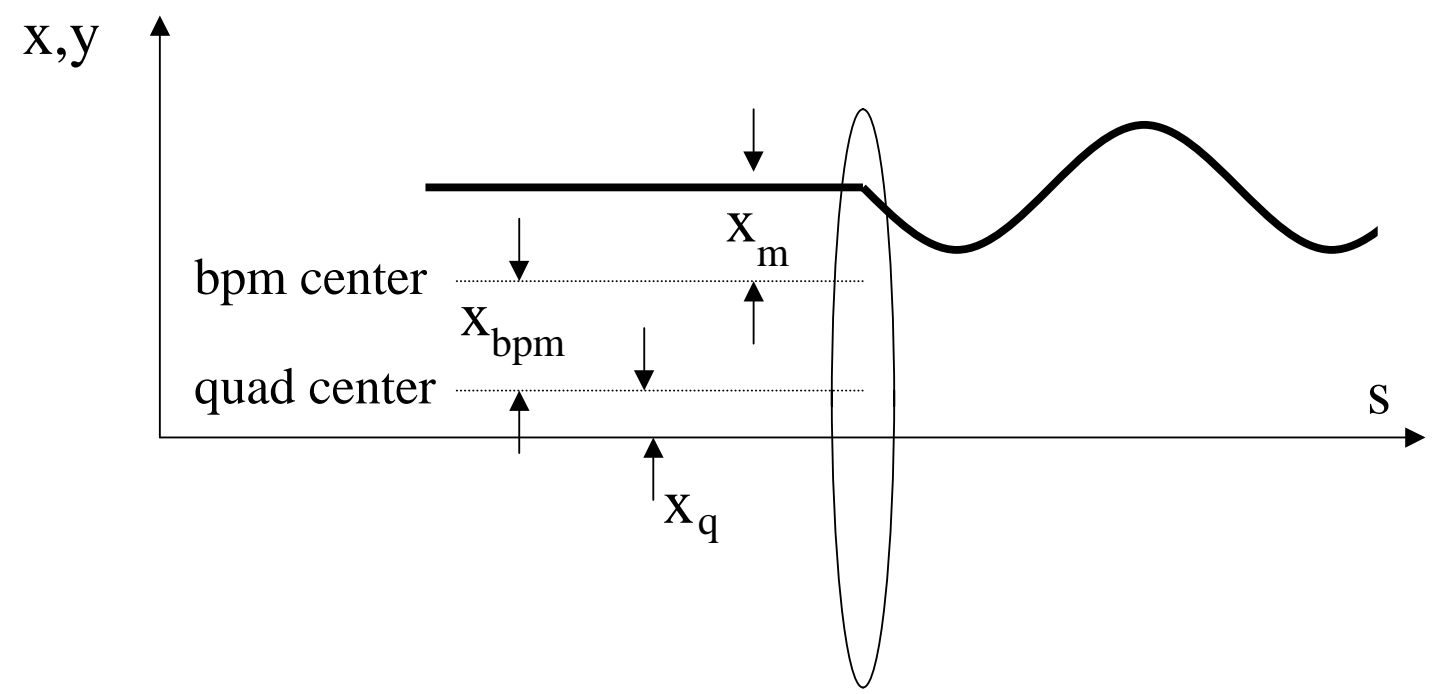

FIGURE 19. Coordinate system used in the example of beam-based alignment. (Courtesy C. Adolphsen (2000).

$$
\left(\begin{array}{c}
x_{k} \\
x_{k}^{\prime} \\
1
\end{array}\right)=R_{j+1, k}\left\{R_{j, j+1}\left[\left(\begin{array}{c}
x \\
x^{\prime} \\
1
\end{array}\right)_{j}+\left(\begin{array}{c}
-x_{q} \\
0 \\
0
\end{array}\right)\right]+\left(\begin{array}{c}
x_{q} \\
0 \\
0
\end{array}\right)\right\}
$$

where ()$_{j}$ gives the beam position and angle with respect to the quad center, the term in [] is the beam position with respect to the reference axis, $R_{j, j+1}[]$ is the beam position with respect to the reference axis transported between quad $j$ and quad $j+1$, and the term in \{\} is the beam position and angle with respect to the quad center transported between quads $j$ and $j+1$. Rearranging terms gives

$$
\left(\begin{array}{c}
x_{k} \\
x_{k}^{\prime} \\
1
\end{array}\right)=R_{0, k}\left(\begin{array}{c}
x \\
x^{\prime} \\
1
\end{array}\right)_{0}+\sum_{j=1}^{k-1}\left(R_{j+1, k}-R_{j, k}\right)\left(\begin{array}{c}
x_{q, j} \\
0 \\
0
\end{array}\right),
$$

where the sum is taken over upstream quadrupoles. The function to be minimized is then

$$
\sum_{k}\left[x_{m}-\left(x_{k}-x_{q}-x_{b p m}\right)\right]^{2}
$$

where $x_{m}$ are the measurements and $\left(x_{k}-x_{q}-x_{b p m}\right)$ is the fitting function with unknowns $x_{q}, x_{b p m}$ and the initial position and angle $x_{0}$ and $x_{0}{ }^{\prime}$.

The number of measurements is about twice the number of unknowns so the system is underconstrained. To constrain the solution, two independent measurements are required. An independent set of data may be obtained by scaling all the quadrupoles and correctors by a common factor and repeating the measurements. Multiple such scalings may be used to overdetermine the system which reduces the sensitivity of the solution to statistical errors. 


\section{C 'Wakefield bumps'}

Through the early 1990's emittance dilutions were controlled by imposing tight tolerances on injection errors as precursor to BNS damping [11,34], steering using both one-to-one correction and localized beam-based alignment $[7,54]$, and by invoking BNS damping. As the beam currents were increased, a more localized emittance preservation technique was developed in which empirically determined trajectory oscillations ('bumps') were used to cancel emittance dilutions from transverse wakefields and dispersive errors. While the origins of the disturbances could not be easily localized longitudinally along the linac, the accumulated effects could be cancelled using such bumps and the emittance dilution could be reduced by a factor of almost ten [55]. The effect of the beam was determined by emittance measurements near the end of the linac (see Appendix B).

Two trajectories in both $x$ and $y$ are shown [55] in Fig. 20. Both trajectories produced about the same small emittance measured near the end of the linac. Notice the vertical scale which shows excursions of nearly $750 \mu \mathrm{m}$ peak-to-peak. While wakefield bumps were used for many years, it became clear as the currents were increased that this technique was inherently unstable; small (e.g. thermal) changes in the reference line phase, for example, changed the phase advance over the bump range so that even this more localized correction scheme was not sufficiently local to be stable against realistic variations in the accelerator. Both trajectories in Fig. 20 resulted in about the same final beam emittances indicating that the procedure was not deterministic.

Physical insights were gained by simulations carried out using the program LIAR [56]. The model included representative amplitudes of the wakefield bumps which minimized the relative emittance growth at the locations of the measurements. The relative growth in normalized emittance is shown $[57,58]$ in Fig. 21 as a function of position along the linac. There are several important conclusions to be drawn from this simulation result:

- Comparison with Fig. 8 shows that at the first emittance measurement, the optimization had been made in a location where the energy spread of the beam was large; that is, a compromise was made using wakefield bumps between correction of dispersive and wakefield-induced errors.

- Between the emittance measurement stations, there was uncontrolled emittance growth.

- Between the final emittance measurement station and extraction of the beam from the linac, there was significant emittance growth.

- Most importantly, being nonlocal in nature, small changes in the phase advance could destroy this delicate cancellation. In practice this caused significant time-dependent variations in the measured emittances [37]- [62]. 

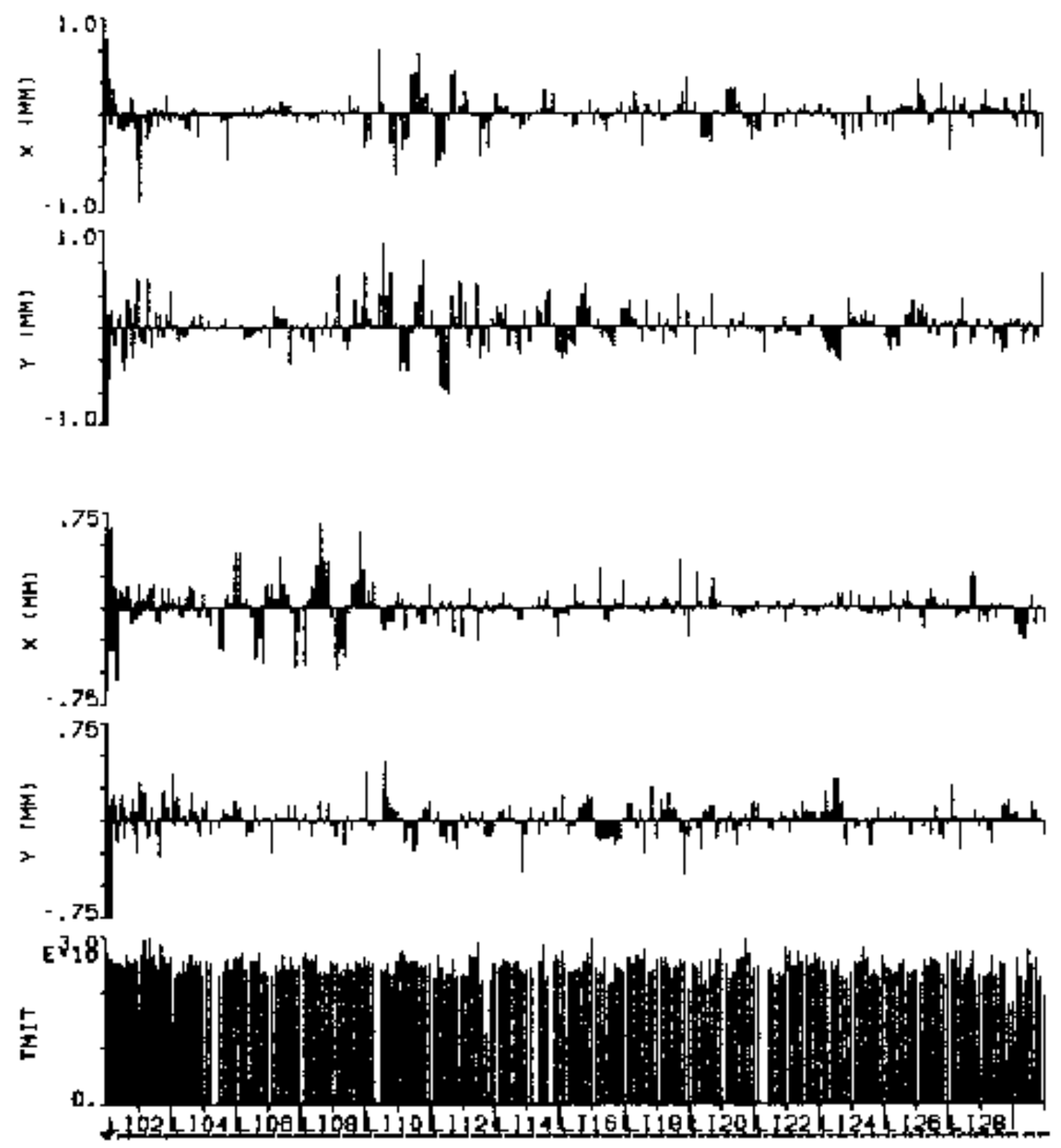

Distance along the linac

FIGURE 20. Two measured orbits with empirically determined coherent betatron oscillations used to cancel accumulated wakefield and dispesion errors. Courtesy J. Seeman (2000). 


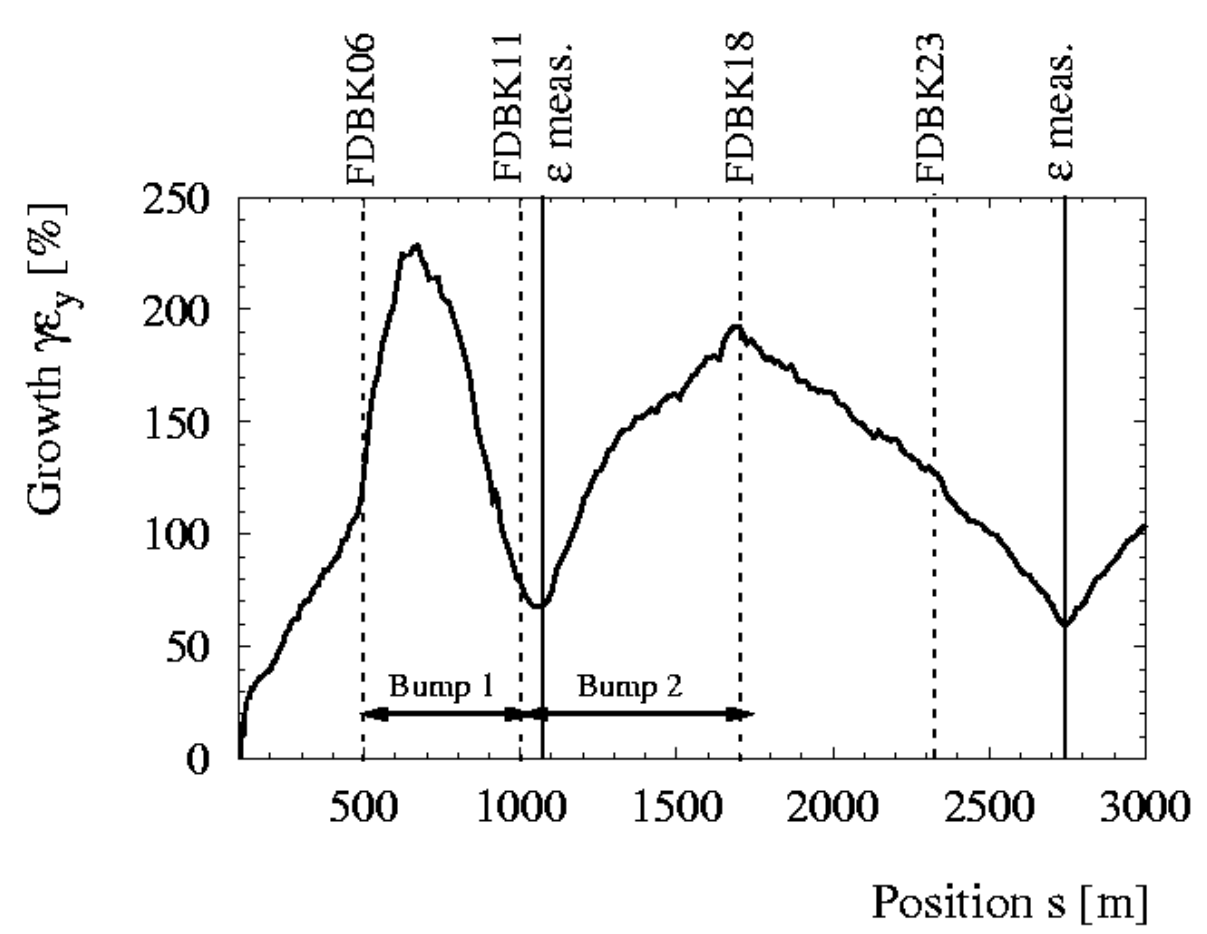

FIGURE 21. Simulated emittance growth as a function of position along the linac. The locations of the feedback loops, which controlled the amplitude of the wakefield bumps, are shown along with the locations of the emittance measurements. Courtesy R. Assmann (2000).

\section{Dispersion-free steering}

So far we have described one-to-one steering which is a first step in orbit optimization but is imperfect as minimization of the BPM reading in a displaced quadrupole generates dispersive errors, beam-based alignment of quadrupole displacements which works beautifully at low beam currents where there is no wakefield-generated dispersion, and wakefield bumps which while more local than BNS damping is highly sensitive to small perturbations in the electromagnetic optics. With perfect implementation of either procedure, dispersive emittance dilutions may still result. As an example, consider a closed trajectory bump of the kind illustrated in Fig. 17. It has been shown [65] using LIAR and realistic optical parameters of the SLC linac that a closed $100 \mu \mathrm{m} \pi$-bump at a quadrupole located early in the linac generates nearly $0.5 \mathrm{~mm}$ dispersion at the end of the linac. Naively, about 6 such bumps acting independently would produce a dispersive emittance contribution equal to the final emittances typically achieved at the SLC.

Dispersion-free steering [63-65] is an algorithm which corrects even more locally dispersive errors from misaligned quadrupoles and dispersive errors arising from transverse wakefields. For mostly technical reasons (e.g. data acquisition and 
processing time) implementation was unduly delayed at the SLC. Dispersion-free steering (and rf phase stability [66-68]) proved crucial for maintaining stable linac emittances at the SLC.

The centroid trajectory ${ }^{5}$ is given by

$$
\begin{aligned}
x_{j} & =\sum_{i=1}^{j-1} \sqrt{\frac{E_{i}}{E_{j}}} \sqrt{\beta_{i} \beta_{j}} \theta_{i} \sin \left(\phi_{j}-\phi_{i}\right) \\
& =\sum_{i=1}^{j-1} R_{12}{ }^{i j} \theta_{i},
\end{aligned}
$$

where the damping factor $\sqrt{\frac{E_{i}}{E_{j}}}$ has been included. To constrain the system, one can equivalently change the beam energy (which is in practice difficult) or as before scale the lattice. Then

$$
\begin{aligned}
\Delta x_{j} & =\sum_{i=1}^{j-1}\left[R_{12}^{i j}-\kappa \sqrt{\frac{E_{i}}{E_{j}}} \sqrt{\beta_{i} \beta_{j}} \sin \left(\phi_{j}-\phi_{i}\right)\right] \theta_{i} \\
& =\sum_{i=1}^{j-1} R_{12, k}^{i j} \theta_{i},
\end{aligned}
$$

where the change in lattice focussing is given by

$$
\kappa=\frac{\Delta K}{K}+1
$$

where $K$ is the quadrupole strength.

The function to be minimized is

$$
\sum_{j}\left[x_{j}-\sum_{i} M_{i j} \theta_{i}\right]^{2}
$$

where $x_{j}$ is an $M \times 1$ vector containing the difference measurements and the fitting function is given by $\sum_{i} M_{i j} \theta_{i}$ where $\theta_{i}$ is an $N \times 1$ vector of unknowns. The $M_{i j}$ represents an $M \times N$ matrix containing the transfer matrix elements.

In practice it is not difficult to minimize not only the difference orbit but simultaneously the absolute orbit. In this case $x_{j}$ is a $2 M \times 1$ vector containing the difference measurements and the absolute orbit, $M_{i j}$ is a $2 M \times N$ matrix and $\theta$ remains an $N \times 1$ matrix. This approach was used at the SLC where in addition, to overconstrain the solution and minimize systematic errors arising from magnet hysteresis, the measurements were performed for 4-5 values of $\kappa$ corresponding to energy variations of $+5 \%$ to $-30 \%$. In later years, the problem of hysteresis was eliminated and the application became noninvasive as two independent measurements could be obtained without changing the lattice by measuring independently the orbits of the electrons and positrons which passed through the same lattice.

5) a reminder: intrabunch position-energy correlations, when projected, may result in measured centroid displacements which underestimate the contributions from off-axis bunch tails 
Shown in Fig. 22 are absolute $(\kappa=1.0)$ and difference trajectories $(\kappa=0.9$, 0.8 , and 0.7) measured after trajectory steering of the SLC linac [65] using 20pulse BPM averaging. With an equivalent energy change of $30 \%(\kappa=0.7)$, a difference trajectory of up to $1.5 \mathrm{~mm}$ was observed. Similar measurements made after iteration of dispersion-free steering [65] are given in Fig. 23. Iteration proved useful to reduce sensitivity to errors in the assumed optics even though experience showed that the first iteration yielded the largest improvements. With $\kappa=0.3$, neglecting the errant point due possibly to a bad BPM near sector 20, the maximum orbit difference after dispersion-free steering was reduced from $1.5 \mathrm{~mm}$ to less than $200 \mu \mathrm{m}$. Notice that the rms of the measurements of the absolute orbit are actually larger following dispersion-free steering. This suggests significant BPM and/or quadrupole misalignment errors.
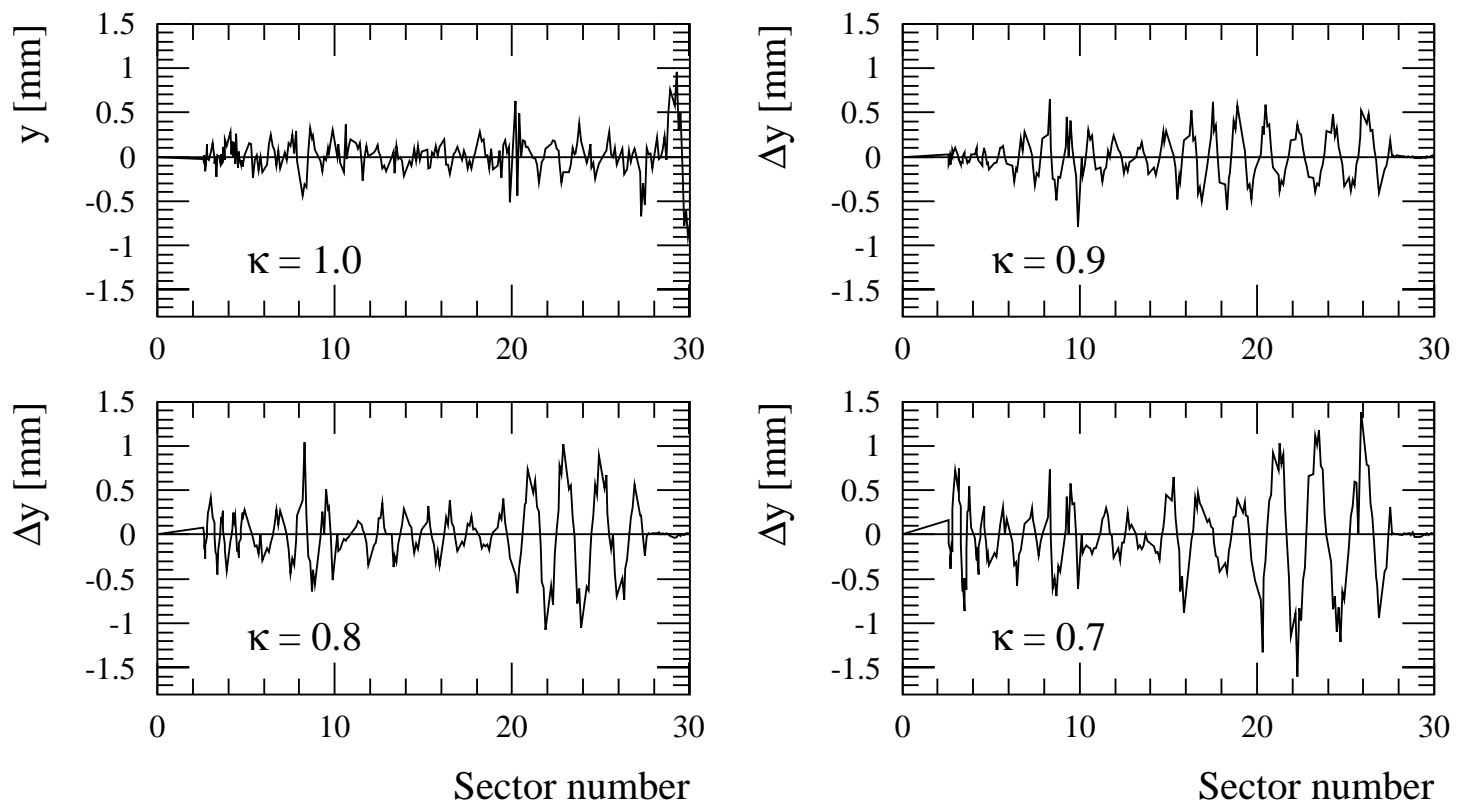

FIGURE 22. Absolute and difference vertical trajectories measured after trajectory steering before dispersion-free steering of the SLC linac. Courtesy R. Assmann (2000).

\section{E Errors}

For simplicity of expression, measurement errors have been neglected up to now. Error sources and their typical rms contributions include BPM resolution errors $\sigma\left(x_{j}\right)<10 \mu \mathrm{m}, \mathrm{bpm}$ misalignments $\sigma_{\mathrm{bpm}} \sim 100 \mu \mathrm{m}$, and systematic errors arising from beam jitter and/or slow drifts $\sigma_{\text {sys }} \sim 20 \mu \mathrm{m}$. To propagate the measurement errors used in the minimization procedures, a weighting function may be defined as 

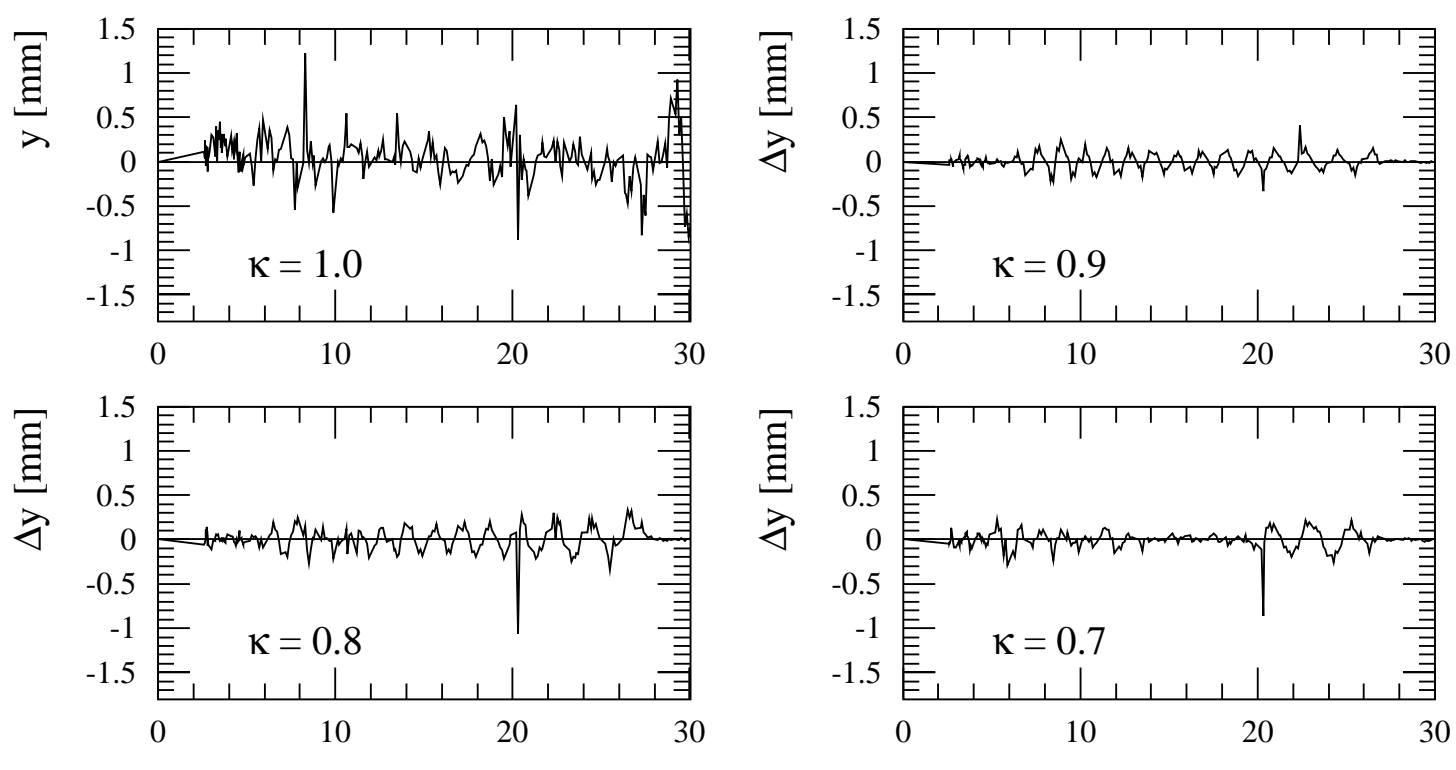

Sector number

Sector number

FIGURE 23. Absolute and difference vertical trajectories measured after dispersion-free steering in the SLC linac. Courtesy R. Assmann (2000).

$$
w_{j}=\frac{1}{\sum_{j} \sigma_{m, j}^{2}},
$$

where the subscripts $m$ give the different error sources and $j$ is a sum over the BPM measurements. The functions to be minimized then are (c.f. Eqs. 38, 42, and 47)

$$
\begin{aligned}
& \sum_{j}\left[\frac{\left.x_{j}-\sum_{i} M_{i j} \theta_{i}\right)}{\sum_{m} \sigma_{m, j}{ }^{2}}\right]^{2}, \quad \text { one-to-one } \\
& \sum_{k}\left[\frac{x_{m}-\left(x_{k}-x_{q}-x_{b p m}\right)}{\sum_{m} \sigma_{m, j}{ }^{2}}\right]^{2}, \text { beam-based alignment } \\
& \sum_{j}\left[\frac{x_{j}-\sum_{i} M_{i j} \theta_{i}}{\sum_{m} \sigma_{m, j}{ }^{2}}\right]^{2}, \quad \text { dispersion-free steering }
\end{aligned}
$$

A goodness of fit parameter, or $\chi$-squared may be correspondingly constructed. In the dispersion-free steering example given above for which both the trajectory and the trajectory differences were to be simultaneously minimized,

$$
\chi^{2}=\sum_{j}\left[\frac{x_{j}^{2}}{\sigma_{\mathrm{bpm}}{ }^{2}}+\sum_{\kappa} \frac{\Delta x_{j, \kappa}{ }^{2}}{\sigma_{\mathrm{sys}}{ }^{2}}\right],
$$

where the second summation over $\kappa$ corresponds to the different energy scalings under which the measurements were made. The errors from BPM resolution were assumed to be negligible and the summation over errors has been simplified to 
reflect the dominating errors; that is, the systematic errors contribute less than the alignment errors in the measurements of the absolute trajectories while in the difference trajectory measurements the BPM misalignments cancel and are therefore set to zero.

\section{CONCLUSION}

In this report we reviewed various mechanisms causing emittance dilution in linear accelerators. We concentrated on those effects which were important for the Stanford Linear Collider. Many experimental measurement techniques were developed and used to suppress emittance growth. In particular powerful procedures were applied to overcome inadequacies in the precision to which the electromagnetic fields governing the beam transport were known. The described methods all contributed to the steady improvements $[1,23,57,69-78]$ in the collider performance, which is summarized [78] in Fig. 24. For the next generation of linear colliders careful control of the beam emittances will be even more important as alignment tolerances are stricter at higher rf frequency. The methods described in this report should be applicable at least during initial commissioning.

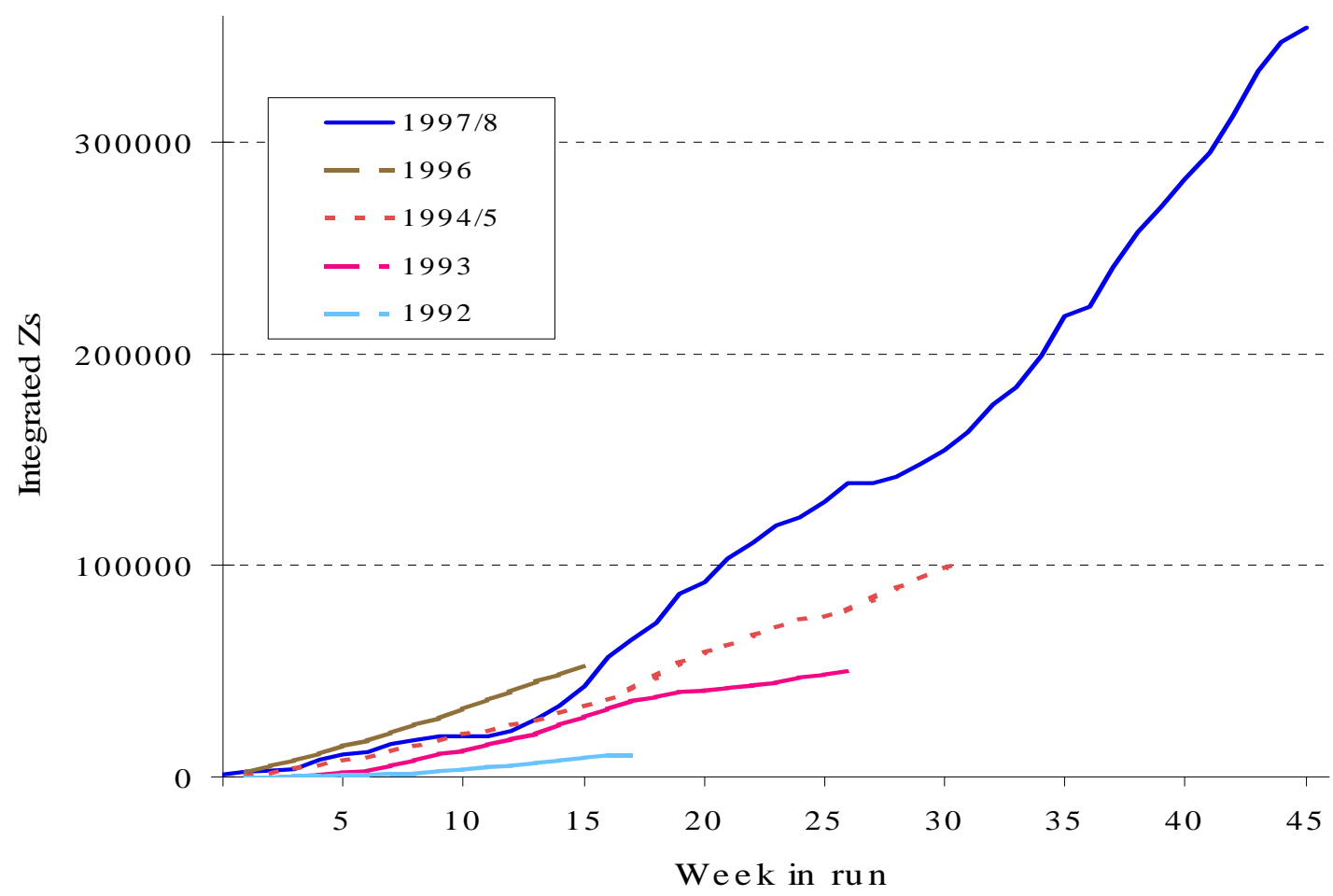

FIGURE 24. History of integrated $Z^{0}$ events recorded at the SLC from 1992 through 1998. Courtesy N. Phinney (2000). 


\section{REFERENCES}

1. J.T. Seeman, 'Status of the Stanford Linear Collider', Proc. ECFA Workshop on $\mathrm{e}^{+} \mathrm{e}^{-}$Linear Colliders (LC92), Garmisch-Partenkirchen, Germany (1992) 93-120.

2. C. Adolphsen, K.L. Bane, J. Seeman, 'Effect of wake fields on first order transport in the SLC linac', Proc. of IEEE Part. Accel. Conf., San Francisco, CA (1991) 32073209 .

3. F.J. Decker et al, 'Dispersion and betatron matching into the linac', Proc. IEEE Part. Accel. Conf., San Francisco, CA (1991) 905-907.

4. L. Merminga P.L. Morton, J. Seeman, W.L. Spence, 'Transverse phase space in the presence of dispersion', Proc. of IEEE Part. Accel. Conf., San Francisco, CA (1991) 461-463.

5. T.L. Lavine et al, 'Beam determination of quadrupole misalignments and beam position monitor biases in the SLC linac', Proc. Lin. Acc. Conf., Williamsburg, VA (1988).

6. J.T. Seeman, 'Linear collider accelerator physics issues regarding alignment', Proc. Acc. Alignment, Stanford, CA (1989) 257-262.

7. C. Adolphsen et al, 'Beam based alignment technique for the SLC linac', Part. Acc. Conf., (1989) 977-979.

8. J.T. Seeman, 'Observation of high current effects in high energy linear colliders', 1990 Joint US-CERN School on Particle Accelerators: Frontiers of Particle Beams, Intensity Limitations, Hilton Head Island, SC (1990); published in CERN US PAS (1990) 255-292.

9. J.T. Seeman et al, 'Alignment issues of the SLC linac accelerating structure', Proc. of Part. Acc. Conf., San Fransisco, CA (1991) 2949-2951.

10. T. Barklow, P. Emma, P. Krejcik, N.J. Walker, 'Review of lattice measurement techniques at the SLC', Proc. 5th ICFA Adv. Beam Dyn. Wkshp., Corpus Christi, TX (1991) 347-354.

11. T. Limberg, J. Seeman, W.L. Spence, 'Effects and tolerances of injection jitter in the SLC and future linear colliders', Proc. of 2nd Eur. Part. Acc. Conf., Nice, France (1990) 1506-1508.

12. F. Rouse et al, 'General, database-driven fast-feedback system for the Stanford Linear Collider', Proc. 1991 Part. Accel. Conf., San Fransisco, CA (1991) 1419-1421.

13. T. Himel et al, 'Use of digital control theory state space formalism for feedback at SLC', Proc. Part. Accel. Conf., San Fransisco, CA (1991) 1451-1453.

14. L. Hendrickson et al, 'Generalized fast feedback system in the SLC', Proc. of the Inter. Conf. on Accel. and Large Experimental Physics Control Systems, Tsukuba, Japan (1991) 414-419.

15. T. Himel et al, 'Adaptive cascaded beam based feedback at the SLC', Proc. 1993 Part. Acc. Conf., Washington, DC (1993) 2106-2108.

16. M.C. Ross, L. Hendrickson, T. Himel, E. Miller, 'Precise system stabilization at SLC using dither techniques', Proc. Part. Acc. Conf., Washington, DC (1993) 1972-1974.

17. L. Hendrickson et al, 'Tutorial on beam-based feedback systems for linacs', Proc. 17th Int. Linear Acc. Conf (LINAC94), Tsukuba, Japan (1994).

18. L. Hendrickson et al, 'Fast feedback for linear colliders', Proc. 16th IEEE Part. Acc. 
Conf., Dallas, TX (1995) 2389-2393.

19. M.G. Minty et al, 'Feedback performance at the Stanford Linear Collider', Proc. 16th IEEE Part. Acc. Conf., Dallas, TX (1995) 662-664.

20. T. Himel, 'Feedback: Theory and accelerator applications', Ann. Rev. Nucl. Part. Sci. 47, (1997) 157-192.

21. H. Schwarz and J.G. Judkins, 'Phase detector and phase feedback for a single bunch in a two bunch damping ring for the SLAC linear collider', Proc. 12th IEEE Part. Acc. Conf., Washington, D.C. (1987) 769-770.

22. C.E. Adolphsen, P.J. Emma, T.H. Fieguth, W.L. Spence, 'Chromatic correction in the SLC bunch length compressors', Proc. of IEEE Part. Accel. Conf., San Francisco, CA (1991) 503-505.

23. J. Seeman and J. Sheppard, 'Status of the SLC', Proc. of 'Orsay Accel. Wkshp. on New Developments in Part. Acc. Techniques', Orsay, France (1987) 122-133.

24. L.Z. Rivkin et al, 'Bunch lengthening in the SLC damping rings', Proc. 1st Eur. Part. Acc. Conf., Rome, Italy (1988).

25. K.L. Bane, 'Bunch lengthening in the SLC damping rings', Impedance and bunch instability workshop adv. phonton source, Argonne, IL (1989); K.L.F. Bane, 'Bunch Lengthening in the SLC Damping Rings', Proc. Acc. Phys. and Modeling, Upton, NY (1991), 235-289.

26. K.L. Bane and R.D. Ruth, 'Bunch lengthening calculations for the SLC damping rings', Part. Accel. Conf., Chicago IL (1989) 789-791.

27. P. Krejcik et al, 'High intensity bunch length instabilities in the SLC damping rings', Proc. Part. Acc. Conf., Washington, DC (1993) 3240-3242.

28. K. Bane et al, 'High intensity single bunch instability behavior in the new SLC damping ring vacuum chamber', Proc. Part. Acc. Conf., Dallas, TX (1995) 31093111.

29. K.L.F. Bane and K. Oide, 'Simulations of the longitudinal instability in the new SLC damping rings', Proc. Part. Acc. Conf., Dallas, TX (1995) 3105-3108.

30. J.T. Seeman, 'The Stanford Linear Collider', Phys. of Part. Acc.. In Jackson, J.D. (ed.) et al: Annual review of nuclear and particle science 41, 389-428.

31. J.T. Seeman, 'Observations and cures of wakefield effects in the SLC Linac', 5th ICFA Adv. Beam Dyn. Wkshp on Effects of Errors in Accelerators, Their Diagnosis and Correction, Corpus Christi, TX (1991) 339-346.

32. C. Adolphsen, T. Slaton, 'Beam trajectory jitter in the SLC linac', Proc. of Part. Acc. Conf., Dallas, TX (1995) 3034-3036.

33. C. Adolphsen et al, 'Pulse to pulse stability issues in the SLC', Proc. of Part. Acc. Conf., Dallas, TX (1995) 645-648.

34. A.W. Chao, B. Richter, C.Y. Yao, 'Beam emittance growth caused by transverse deflecting fields in a linear accelerator', Nucl. Instrum. Meth. 178, (1980) 1-24.

35. A.W. Chao, B. Richter, C.Y. Yao, 'Transverse wake field effects on intense bunches with application to the SLAC linear accelerator', Proc. XI Intl. Conf. on High Energy Acc., Geneva, Switzerland (1980) 597-604.

36. J.T. Seeman, K.L. Bane, T. Himel, W.L. Spence, 'Observation and control of emittance growth in the SLC linac', Part. Accel., 30, (1989) 97-104.

37. F.J. Decker, R. Brown, J.T. Seeman, 'Beam size measurements with noninterceptive 
off-axis screens', Proc. of Part. Acc. Conf., Washington, DC (1993) 2507-2509.

38. A.W. Chao, (ed.) and M. Tigner, (ed.), 'Handbook of accelerator physics and engineering', Singapore, Singapore: World Scientific (1999) 650 p.

39. P.B. Wilson, 'A study of beam blow-up in electron linacs', Report No. HEPL-297 (Rev. A), High Energy Physics Laboratory, Stanford University, Stanford, California (1963).

40. R.B. Neal and W.K.H. Panofsky, Science 152,(1966) 1353.

41. W.K.H. Panofsky and M. Bander, 'Asymptotic theory of beam break-up in linear accelerators', Rev. Sci. Instr. 39, 206 (1968).

42. G.V. Stupakov, 'BNS damping of beam breakup instability', SLAC-AP-108 (1997).

43. V. Balakin, S. Novokhatsky, V. Smirnov, 'VLEPP: transverse beam dynamics' Proc. 12th Int. Conf. on High Energy Acc. (1983) 119-120.

44. J.T. Seeman, F.J. Decker, R.L. Holtzapple, W.L. Spence, 'Measured optimum BNS damping configuration of the SLC linac', Proc. Part. Acc. Conf., Washington, DC (1993) 3234-3236.

45. J.T. Seeman and N. Merminga, 'Mutual compensation of wakefield and chromatic effects of intense linac bunches', Proc. 1990 Linac Conf., Albuquerque, NM (1990) 387-389.

46. K.L.F. Bane et al, 'Measurement of the londitudinal wake field and the bunch shape in the SLAC linac', Proc. 1997 Part. Acc. Conf., Vancouver, BC, Canada (1997).

47. C. Adolphsen, K.L. Bane, J. Seeman, 'Effect of wake fields on first order transport in the SLC linac', Proc. of Part. Acc. Conf., San Fransisco, CA (1991) 3207-3209.

48. R.L. Holtzapple, 'Longitudinal dynamics at the Stanford Linear Collider', PhD thesis, (June, 1996); R.L. Holtzapple et al, 'Measurements of longitudinal phase space in the SLC linac', Proc. Part. Acc. Conf., Dallas, TX (1995) 3025-3027.

49. R.L Holtzapple, 'Bunch compression at the Stanford Linear Collider', Proc. of Micro Bunches: A Workshop on the Production, Measurement and Applications of Short Bunches of Electrons and Positrons in Linacs and Storage Ringe, Upton, NY (1995) $36-45$.

50. F.J. Decker et al, 'Longitudinal phase space setup for the SLC beams', Proc. Part. Acc. Conf., Vancouver, BC, Canada (1997) 509-511.

51. G.A. Loew and J.W. Wang, 'Minimizing the energy spread within a single bunch by shaping its charge distribution', Proc. Part. Acc. Conf., Vancouver, BC Canada (1985) 3228-3230.

52. F.J. Decker, R Holtzapple, T. Raubenheimer, 'Overcompression, a method to shape the longitudinal bunch distribution for a reduced energy spread', Proc. 17th Intl. Linear Acc. Conf., Tsukuba, Japan (1994), 47-49.

53. K. Thompson et al, 'Operational experience with model based steering in the SLC linac', Proc. Part. Acc. Conf., Chicago, IL (1989), 1675-1677.

54. P. Emma, T.H. Fieguth, T. Lohse, 'Online monitoring of dispersion functions and transfer matrices at the SLC', Nucl. Instrum. Meth. A 288, (1990) 313-334.

55. J.T. Seeman, F.J. Decker, I. Hsu, 'The introduction of trajectory oscillations to reduce emittance growth in the SLC linac,', Proc. 15th Intl. Conf. High Energy Acc., Hamburg, Germany (1992) 879-881.

56. R. Assmann et al, 'LIAR - A computer program for the modeling and simulation of 
high performance linacs', SLAC/AP-103, 1997.

57. R. Assmann, 'Beam dynamics in the SLC', Proc. of Part. Acc. Conf., Vanouver, BC, Canada (1997).

58. R. Assmann, F.J. Decker, P. Raimondi, 'Improvements in emittance wakefield optimization for the SLAC linear collider', Proc. of 6th Eur. Part. Acc. Conf., Stockholm, Sweden (1998).

59. F.J. Decker et al, 'Diganostic beam pulses for monitoring the SLC linac', Proc. 16th Part. Acc. Conf., Dallas TX (1995) 2646-2648.

60. C. Adolphsen et al, 'Collective centroid oscillations as an emittance preservation diagnostic in linear collider linacs', Proc. 17th Part. Acc. Conf., Vancouver, Canada (1997).

61. F.J. Decker et al, 'Beam based analysis of day night performance variations at the SLC linac', Proc. 17th Part. Acc. Conf., Vancouver, Canada (1997) 506-508.

62. R.W. Assmann et al, 'Beam based monitoring of the SLC linac optics with a diagnostic pulse', Proc. 17th Part. Acc. Conf., Vancouver, Canada (1997) 497-499.

63. T.O. Raubenheimer and R.D. Ruth, 'A dispersion free trajectory correction technique for linear colliders', Nucl. Instr. and Meth. A302, (1991) 191-208.

64. R. Assmann et al, 'Quadrupole alignment and trajectory correction for future linear colliders: SLC tests of a dispersion-free steering algorithm', Proc. 4th Int. Workshop on Acc. Alignment, Tsukuba, Japan (1995).

65. R. Assmann, T. Chen, F.J. Decker, M. Minty, P. Raimondi, T.O. Raubenheimer, R. Siemann, 'Simultaneous trajectory and dispersion correction in the SLC linac' (1996), unpublished.

66. D. McCormick, M. Ross, T. Himel, N. Spencer, 'Thermal stabilization of low level RF distribution systems at SLAC', Proc. of Part. Acc. Conf., Washington, DC (1993) 1975-1977.

67. F.J. Decker et al, 'Effects of temperature variation on the SLC linac rf system', Proc. of Part. Acc. Conf., Dallas, TX (1995) 1821-1823.

68. J. Bogart et al, 'A fast and accurate phasing algorithm for the rf accelerating voltages of the SLAC linac', Proc. 6th Eur. Part. Acc. Conf. Stockholm, Sweden (1998) 22-26.

69. G.S. Abrams et al, 'Experimental beam dynamics and stability in the SLC linac', Proc. 14th Int. Conf. on High Energy Acc., Tsukuba, Japan (1989); Part. Accel. 30, (1990) 91-96..

70. J.L. Turner et al, 'Vibration studies of the stanford linear accelerator', Proc. of Part. Acc. Conf., Dallas, TX (1995) 665-667.

71. F. Zimmermann et al, 'Performance of the 1994/1995 SLC final focus system', Proc. of Part. Acc. Conf., Dallas, TX (1995) 656-658.

72. P. Emma, 'The Stanford Linear Collider', Proc. of Part. Acc. Conf., Dallas TX (1995) 606-610.

73. P. Emma, L.J. Hendrickson, P. Raimondi, F. Zimmermann, 'Limitations of interaction point spot size tuning at the SLC', Proc. of Part. Acc. Conf., Vancouver, Canada (1997) 452-454.

74. R.W. Assman et al, 'Accelerator physics highlights in the 1997/98 SLC run', Proc. 1st Asian Part. Acc. Conf., Tsukuba, Japan (1998) 474-476.

75. F. Zimmermann, '1998 SLC luminosity and pinch enhancement', SLAC-CN-418 
(1998) 7pp.

76. F.J. Decker, M.G. Minty, Y. Nosochkov, P. Raimondi, 'Status of the SLC linac', Proc. Eur. Part. Acc. Conf. (1998).

77. R.W. Assmann et al, 'Accelerator physics highlights in the 1997/98 SLC run', Proc. 1st Asian Part. Acc. Conf. (1998) Tsukuba, Japan.

78. P. Raimondi et al, 'Recent luminosity improvements at the SLC', Proc. 6th Eur. Part. Acc. Conf. Stockholm, Sweden (1998).

79. D.A. Edwards and M.J. Syphers, 'An introduction to the physics of high energy accelerators', Wiley series in beam physics and accelerator technology, New York, USA (1993).

80. E.D. Courant and H.S. Snyder, "Theory of the alternating-gradient synchrotron", Annals of Physics 3, (1958) 1-48.

81. K.L. Brown, 'A first and second-order matrix theory for the design of beam transport systems and charged particle spectrometers", SLAC-75 (1982).

82. K.L. Brown, F. Rothacker, D. Carey, C. Iselin, 'Transport. A computer program for designing charged particle beam transport systems", SLAC-91 (1977).

83. H. Wiedemann, 'Particle accelerator physics: basic principles and linear beam dynamics', Springer Verlag, Berlin, Germany (1993).

84. M.C. Ross et al, 'Automated emittance measurements in the SLC', Proc. of Part. Acc. Conf., Washington, DC (1989) 725-728.

\section{Appendix A - Definition of the Beam Emittance}

The reader is refered to Ref. [79]. The beam emittance $\epsilon$ describes the phase space area occupied by the beam. For a Gaussian beam with standard deviation $\sigma$, the phase space area containing a fraction $F$ of the beam is

$$
\epsilon=-\frac{2 \pi \sigma^{2}}{\beta} \ln (1-F)
$$

where $\beta$ is the $\beta$-function at the observation point (the Twiss parameters $\alpha, \beta$, and $\gamma$ are described in numerous texts such as in Refs. [80]- [83]). Various definitions, which depend on the choice of $F$, are used depending on the particular application. In this report, we take $F=15 \%$ so that

$$
\epsilon=\frac{\sigma^{2}}{\beta}
$$

The standard deviation $\sigma$ is often taken to represent the root-mean-square (rms) of the distribution. It is given by

$$
\sigma_{x}=\sqrt{\left.<x^{2}>-<x\right\rangle^{2}}
$$

where $x$ represents either the horizontal or the vertical plane. Here $\langle x\rangle$ and $<x^{2}>$ are the first and second moment of the beam distribution, respectively. For an intensity distribution $f(x)$, 


$$
\begin{aligned}
<x> & =\frac{\int_{0}^{\infty} x f(x) d x}{\int_{0}^{\infty} f(x) d x} \\
<x^{2}> & =\frac{\int_{0}^{\infty} x^{2} f(x) d x}{\int_{0}^{\infty} f(x) d x}
\end{aligned}
$$

Often the physical quantity of interest is given by Eq. 53 with the static position offset of the beam intensity centroid omitted so that

$$
\epsilon=\frac{<x^{2}>^{\frac{1}{2}}}{\beta} .
$$

\section{Appendix B - Measurements of the Beam Emittance}

The transformation between an initial beam matrix $\sigma_{0}$ and the beam matrix $\sigma$ at a desired observation point is given by

$$
\sigma=M \sigma_{0} M^{T}
$$

where the beam matrix, in terms of the Twiss parameters ( [80]- [83]), is

$$
\sigma=\epsilon\left(\begin{array}{cc}
\beta & -\alpha \\
-\alpha & \gamma
\end{array}\right)
$$

In an uncoupled system,

$$
\sigma=\left(\begin{array}{cccc}
\sigma_{11} & \sigma_{12} & 0 & 0 \\
\sigma_{21} & \sigma_{22} & 0 & 0 \\
0 & 0 & \sigma_{33} & \sigma_{34} \\
0 & 0 & \sigma_{43} & \sigma_{44}
\end{array}\right) \text { and } M=\left(\begin{array}{cccc}
M_{11} & M_{12} & 0 & 0 \\
M_{21} & M_{22} & 0 & 0 \\
0 & 0 & M_{33} & M_{34} \\
0 & 0 & M_{43} & M_{44}
\end{array}\right)
$$

The beam matrix is symmetric with $\sigma_{12}=\sigma_{21}$, but in general $M_{12} \neq M_{21}$.

Single wire measurement of the beam emittance [84]

An (invasive) measurement of the beam emittance can be made by varying the field strength of a quadrupole located upstream of a single wire or screen. The transfer matrix is $M=S Q$, where $S$ is the transfer matrix of the quadrupole:

$$
Q=\left(\begin{array}{cc}
1 & 0 \\
k= \pm \frac{1}{f} & 1
\end{array}\right)
$$

using a thin-lens approximation for which the length of the quadrupole is short compared to it's focal length $f$. After mutiplying matrices,

$$
M=\left(\begin{array}{ll}
S_{11}+k S_{12} & S_{12} \\
S_{21}+k S_{22} & S_{22}
\end{array}\right) .
$$


Expanding the matrix product $\sigma=(S Q) \sigma_{0}(S Q)^{T}$ and equating the (11) element on both sides, the beam size is

$$
\sigma_{11}=\left(S_{11}^{2} \sigma_{110}+2 S_{11} \sigma_{120}+S_{12}^{2} \sigma_{220}\right)+\left(2 S_{11} S_{12} \sigma_{110}+2 S_{12}^{2} \sigma_{120}\right) k+S_{12}^{2} \sigma_{11} k^{2},
$$

which is quadratic in the field parameter $k$.

\section{Procedure (for a single-wire wire scanner measurement)}

1. For each value of quadrupole field strength $k$, scan the wire to obtain detector counts as a function of wire position.

2. For each wire scan at fixed $k$, fit the measured distribution to a Gaussian of the form

$$
f(x)=f_{0}+f_{\max } e^{-\frac{(x-<x>)^{2}}{2<x^{2}>}},
$$

where $f_{0}$ is the basline level offset and $f_{\max }$ is the peak value of the Gaussian distribution.

3. Plot the fitted $\left\langle x^{2}\right\rangle$ as a function of $k$.

4. Fit this result to a parabola. One parametrization for the fit is

$$
\begin{aligned}
\sigma_{11} & =A(k-B)^{2}+C \\
& =A k^{2}-2 A B k+(C+A B)^{2} .
\end{aligned}
$$

5. Reconstruct the $\sigma$ matrix by equating coefficients:

$$
\begin{gathered}
A=S_{12}^{2} \sigma_{11} \\
-2 A B=2 S_{11} S_{12} \sigma_{11}+2 S_{12}^{2} \sigma_{12} \\
C+A B=S_{11}^{2} \sigma_{11}+2 S_{11} S_{12} \sigma_{12}+S_{12}^{2} \sigma_{22}
\end{gathered}
$$

and solve for $\sigma_{11}, \sigma_{12}\left(=\sigma_{21}\right)$, and $\sigma_{22}$. The results are

$$
\begin{gathered}
\sigma_{11}=\frac{A}{S_{12}^{2}} \\
\sigma_{12}=-\frac{A}{S_{12}^{2}}\left(B+\frac{S_{11}}{S_{12}}\right), \\
\sigma_{22}=\frac{1}{S_{12}^{2}}\left[\left(A B^{2}+C\right)+2 A B\left(\frac{S_{11}}{S_{12}}\right)+A\left(\frac{S_{11}}{S_{12}}\right)^{2}\right]
\end{gathered}
$$

6. Calculate the beam emittance from the determinant of the beam matrix $\epsilon=$ $\sqrt{\operatorname{det} \sigma}$ and propagate errors:

$$
\begin{aligned}
\operatorname{det} \sigma & =\sigma_{11} \sigma_{22}-\sigma_{12}^{2} \\
& =\frac{A C}{S_{12}^{4}} \text { so } \quad \epsilon=\frac{\sqrt{A C}}{S_{12}^{2}} .
\end{aligned}
$$


One can also obtain the ellipse parameters $\alpha, \beta$, and $\gamma$ :

$$
\begin{aligned}
\beta & =\frac{\sigma_{11}}{\epsilon}=\sqrt{\frac{A}{C}} \\
\alpha=-\frac{\sigma_{12}}{\epsilon} & =\sqrt{\frac{A}{C}}\left(B+\frac{S_{11}}{S_{12}}\right) \\
\gamma=\frac{1}{\epsilon} & =\frac{S_{12}^{2}}{\sqrt{A C}}\left[\left(A B^{2}+C\right)+2 A B \frac{S_{11}}{S_{12}}+A\left(\frac{S_{11}}{S_{12}}\right)^{2}\right] .
\end{aligned}
$$

As a check, the ellipse parameters should satisfy $\beta \gamma-1=\alpha^{2}$.

An example emittance measurement made in two transverse planes is shown in Fig. 25. The graphics output shows the square of the measured beam size in $\mu m^{2}$ as a function of the quadrupole field strength in $\frac{\mathrm{kG}}{m} m$. The first two rows of text show the measured emittance $(\epsilon)$ and the normalized emittance $(\gamma \epsilon)$.

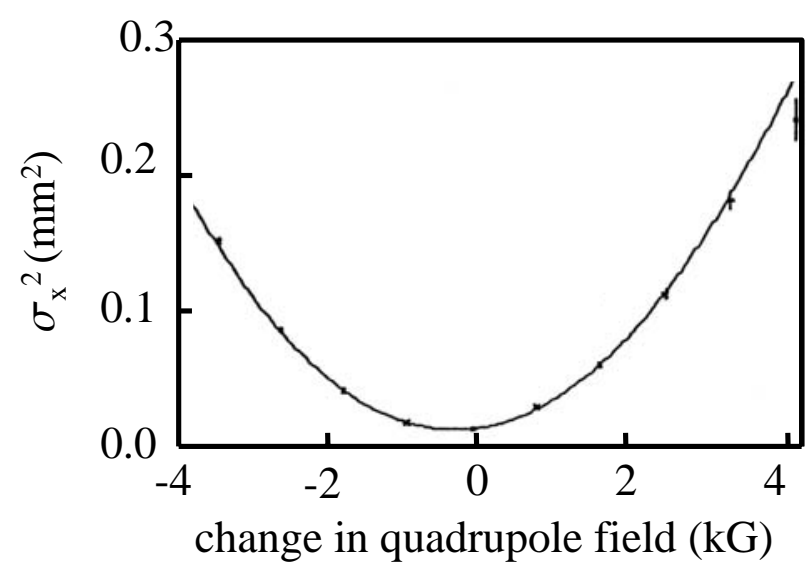

$\epsilon_{\mathrm{x}}=12.9 \pm 0.2 \mathrm{~nm}-\mathrm{rad}$ $\gamma \epsilon_{\mathrm{x}}=30.1 \pm 0.4 \mu \mathrm{m}-\mathrm{rad}$

$$
\begin{aligned}
& \mathrm{A}=3494 \pm 52 \\
& \mathrm{~B}=-118.8 \pm 0.03 \\
& \mathrm{C}=3.2 \mathrm{E} 4 \pm 297 \\
& \chi^{2} / \mathrm{DOF}=1.5
\end{aligned}
$$

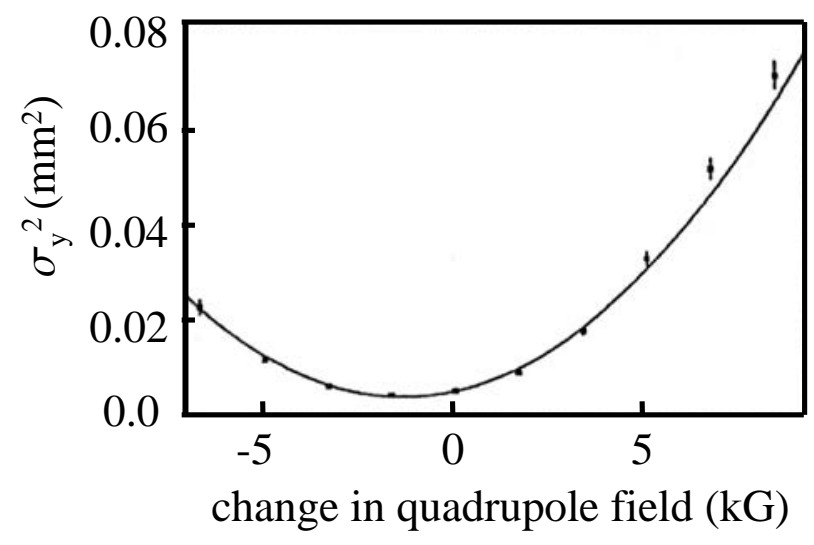

$\epsilon_{\mathrm{y}}=1.71 \pm 0.02 \mathrm{~nm}-\mathrm{rad}$ $\gamma \epsilon_{\mathrm{y}}=3.98 \pm 0.04 \mu \mathrm{m}-\mathrm{rad}$

$$
\begin{aligned}
& \mathrm{A}=158.5 \pm 3.5 \\
& \mathrm{~B}=-129.3 \pm 0.08
\end{aligned}
$$$$
\mathrm{C}=5.1 \mathrm{E} 3 \pm 103
$$$$
\chi^{2} / \mathrm{DOF}=4.6
$$

FIGURE 25. Transverse beam emittance measurements from the SLC made using a quadrupole scan and a single wire. 


\section{Multiple wire measurement of the beam emittance [83]}

The beam emittance may be measured (in many applications noninvasively) using a minimum of 3 wires if there are no coupling elements or using 4 wires with coupling. The optimum wire locations for maximum sensitivity are such that the separation between wires corresponds to a difference in betatron phase advance $\Delta \phi$ of $\frac{90^{\circ}}{N_{w}}$, where $N_{w}$ is the number of wires used in the measurement. Letting $\sigma_{i}$ denote the measured $\sigma_{11}$ 's for wire $i$, and considering the case of 4 wires, the matrix equation to be solved is

$$
\left(\begin{array}{l}
\sigma_{1} \\
\sigma_{2} \\
\sigma_{3} \\
\sigma_{4}
\end{array}\right)=\left(\begin{array}{lll}
c_{1}^{2} & 2 c_{1} s_{1} & s_{1}^{2} \\
c_{2}^{2} & 2 c_{3} s_{2} & s_{2}^{2} \\
c_{3}^{2} & 2 c_{3} s_{3} & s_{3}^{2} \\
c_{4}^{2} & 2 c_{4} s_{4} & s_{4}^{2}
\end{array}\right)\left(\begin{array}{l}
\sigma_{11} \\
\sigma_{12} \\
\sigma_{22}
\end{array}\right)
$$

where $c_{k}$ and $s_{k}$ are elements of the point-to-point transformation matrix from the reference point $\left(x_{0}, x_{0}{ }^{\prime}\right)$ and the location of the wire $\left(x_{k}, x_{k}{ }^{\prime}\right)$; i.e. $x_{k}=c_{k} x_{0}+s_{k} x_{0}{ }^{\prime}$. Notice that $M$ need not be a square matrix. Rewriting Eq. 70 as $A=M C$, then $M^{T} A=M^{T} M C$, or $C=\left(M^{T} M\right)^{-1} M^{T} A$; that is,

$$
\left(\begin{array}{l}
\sigma_{11} \\
\sigma_{12} \\
\sigma_{22}
\end{array}\right)=\left(M^{T} M\right)^{-1} M^{T}\left(\begin{array}{l}
\sigma_{1} \\
\sigma_{2} \\
\sigma_{3} \\
\sigma_{4}
\end{array}\right)
$$

which gives the beam matrix elements $\left(\sigma_{i j}\right)$ in terms of the measured sigmas.

\section{Procedure (for multiple-wire wire scanner measurement)}

1. Scan each wire to obtain detector counts as a function of wire position.

2. For each wire scan, fit the distribution to a Gaussian function using Eq. 62.

3. Reconstruct the $\sigma$ matrix using Eq. 62, the transfer matrix elements $M_{i}$ from the model, and the $\sigma_{i}$ from the measurements.

4. Calculate the emittance $\epsilon=\sqrt{\operatorname{det} \sigma}$.

5. Calculate the ellipse parameters $\alpha=-\frac{\sigma_{12}}{\epsilon}, \beta=\frac{\sigma_{11}}{\epsilon}$, and $\gamma=\frac{\sigma_{22}}{\epsilon}$.

\section{Graphics}

Increased tuning efficiency may be obtained from meaningful graphical representation of the experimental data. In the multiple wire emittance measurement it is useful to project the measurements to a single point along the accelerator and to plot the normalized phase space. Defined in terms of the ellipse parameters, the emittance is

$$
\epsilon=\gamma x^{2}+2 \alpha x x^{\prime}+\beta x^{2}
$$

Since $\beta \gamma=1+\alpha^{2}$, 


$$
\begin{aligned}
\epsilon & =\frac{1}{\beta}\left[x^{2}+\left(\alpha x+\beta x^{\prime}\right)^{2}\right] \\
& =\frac{1}{\beta}\left(x+p_{x}^{2}\right),
\end{aligned}
$$

where $p_{x}=\alpha x+\beta x^{\prime}$ is the canonically conjugate coordinate to $x$.

Procedure (for graphical representation of the emittance measurement) 1. Plot the design rms ellipse in the phase space (a circle)

$$
\left(\frac{x}{\sqrt{\beta}}, \frac{\alpha x+\beta x^{\prime}}{\sqrt{\beta}}\right)
$$

at some reference point $s$ along the trajectory. Normalize the design ellipse to unit radius.

2. In the same figure, plot the ellipse obtained from the measurements of the ellipse parameters at the reference point. Apply the same normalization as in step 1.

3. Using the model of for the lattice, for each wire project its orientation back to the reference point and add the result to the figure; that is, for each point along the wire $\left(x, x^{\prime}\right)_{w}$, do an inverse mapping to the reference point

$$
\left(\begin{array}{c}
x \\
x^{\prime}
\end{array}\right)_{\text {ref pt }}=M^{T}\left(\begin{array}{c}
x \\
x^{\prime}
\end{array}\right)_{w} .
$$

The display should summarize the measurements which might include the measured and expected beam widths at each of the wires, the measured and design beam emittances, and the beam intensity.

An example of such graphics from measurements at the SLC is shown in Fig. 26. From Fig. 26 it is immediately obvious that while the measured ellipse has roughly the same area as the design circle, the orientation of the ellipse is incorrect. From the figure, can be immediately deduced the degree of phase space coverage spanned by the wires. In the horizontal plane, for example, the wire orientations are about $0^{\circ},-45^{\circ},-22.5^{\circ}$, and $-67.5^{\circ}$, which is ideal for the 4 -wire measurement.

The 'measured ellipse', that is the ellipse that was reconstructed from the individual wire scans based on the measured beam widths and the model-dependent transport matrices, does not in this specially selected case represent the true rms distribution of the beam. The raw data used in this measurement are given in Fig. 27. For these complex particle distributions a better characterization of the rms was obtained using an 'asymmetric Gaussian' distribution fucntion for which the left and right hand sides of the measured beam profile were independently fit with two separate Gaussian functions. The fitting function used was

$$
f(x)=f_{0}+f_{\text {max }} e^{-\frac{(x-<x>)^{2}}{2<x^{2}>(1+\alpha[\operatorname{sign}(x-<x>)]}}
$$

where $\alpha$ represents an asymmetry factor and is zero for a perfectly Gaussian beam distribution. The $\sigma$ for the left and right hand sides of the fitted distribution are 

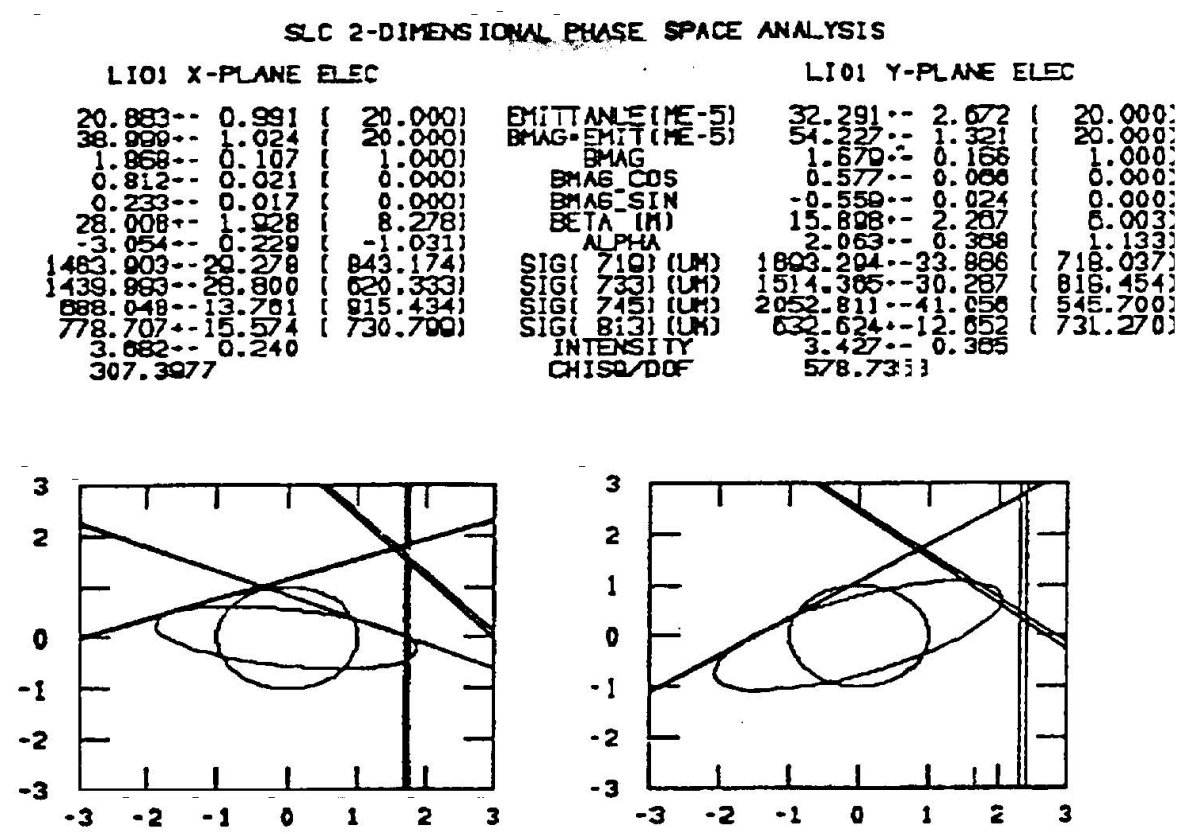

FIGURE 26. Transverse beam emittance measurements from the SLC injector made using multiple wires.

$\sigma=<x^{2}>(1 \pm \alpha)$. For the ellipse reconstruction the average $\sigma$ was used. When large tails are present in the raw data this more accurately represents the beam distribution. Based on the raw data however it is clear that even with the modified fitting algorithm, the fit only marginally represents the actual beam distributions.

For reasonably well 'matched' beams, the graphical summary display is most useful. In this example however, the raw data are more revealing: the double-humps in the raw data are characteristic of an upstream error: a beam, if kicked transversely will filament (lose coherency due to the natural spread in betatron phase advance) resulting in such double-humps and an increased projected emittance.

If a wire is mounted at $45^{\circ}$ with respect to $x$ and $y$, then it is also possible to measure the coupling between $x$ and $y$. The full $\sigma$-matrix is

$$
\left(\begin{array}{llll}
\sigma_{11} & \sigma_{12} & \sigma_{13} & \sigma_{14} \\
\sigma_{21} & \sigma_{22} & \sigma_{23} & \sigma_{24} \\
\sigma_{31} & \sigma_{32} & \sigma_{33} & \sigma_{34} \\
\sigma_{41} & \sigma_{42} & \sigma_{43} & \sigma_{44}
\end{array}\right)
$$

where for example $\sigma_{14}$ represents the coupling between $x$ and $y^{\prime}$. Notice that $\sigma_{14} \neq \sigma_{23}$ so that, whereas for the single plane the uncoupled beam matrix reconstruction required a minimum of 3 measurements, to fully reconstruct the coupled beam matrix a total of at least 10 measurements are needed. This includes 3 measurements in the $x$ plane, 3 measurements in the $y$ plane, and 4 measurements in the $u$ plane. An example of a coupled emittance measurement is presented in Figs. 
28-31. In this case the raw data are well fit using a Gaussian function. In the text of Fig. 28 the parameters $\epsilon_{1}$ and $\epsilon_{2}$ represent the emittance one would measure in the absence of coupling. They are in good agreement with the measured emittances $\epsilon_{x}$ and $\epsilon_{y}$.
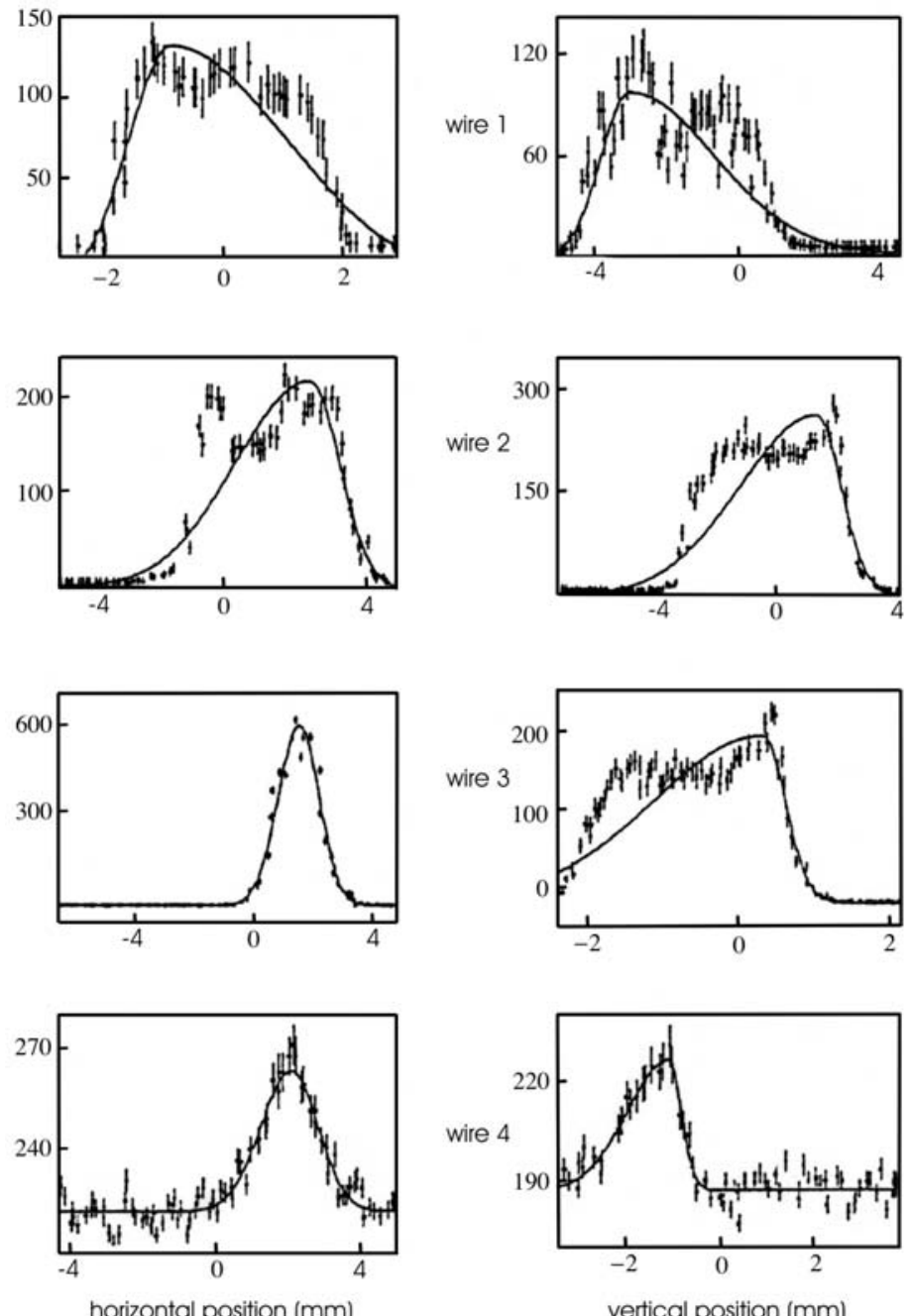

vertical position $(\mathrm{mm})$

FIGURE 27. Raw data showing individual wire scans used in the emittance measurement summarized in Fig. 26 and 'asymmetric' Gaussian fits (c.f. Eq.76). 

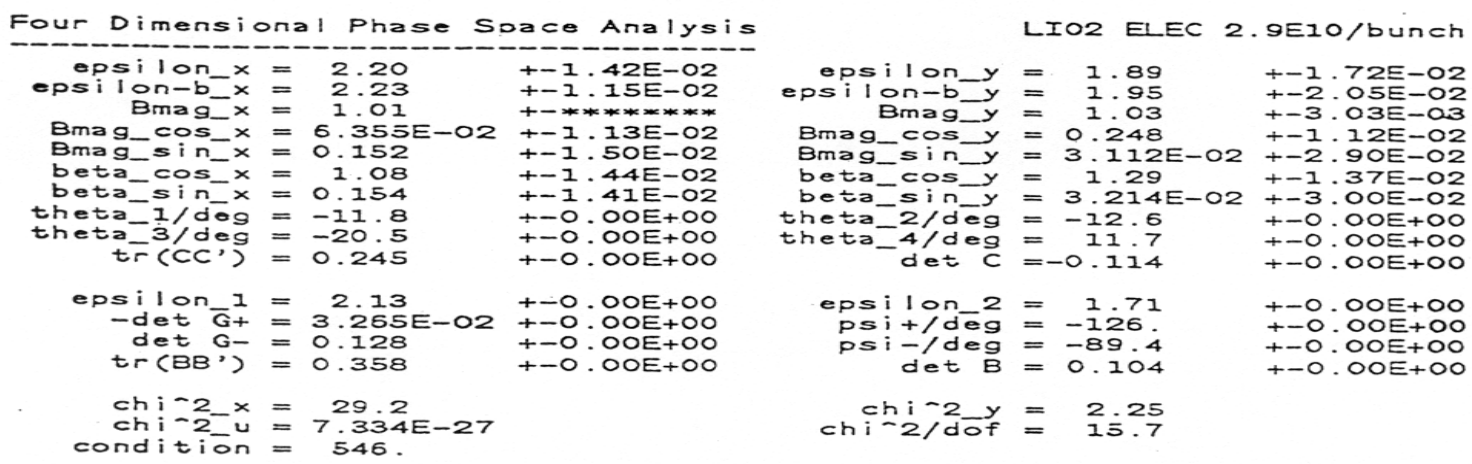

FIGURE 28. Summary display for a 4-dimensional emittance measurement in the SLC linac.
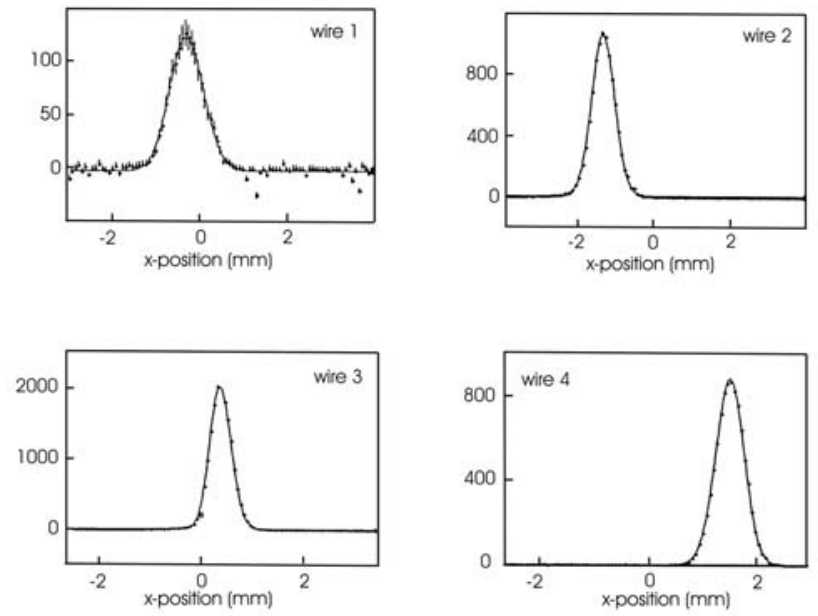

FIGURE 29. Raw $x$-plane data corresponding to the 4-dimensional emittance measurement summarized in Fig. 28. 

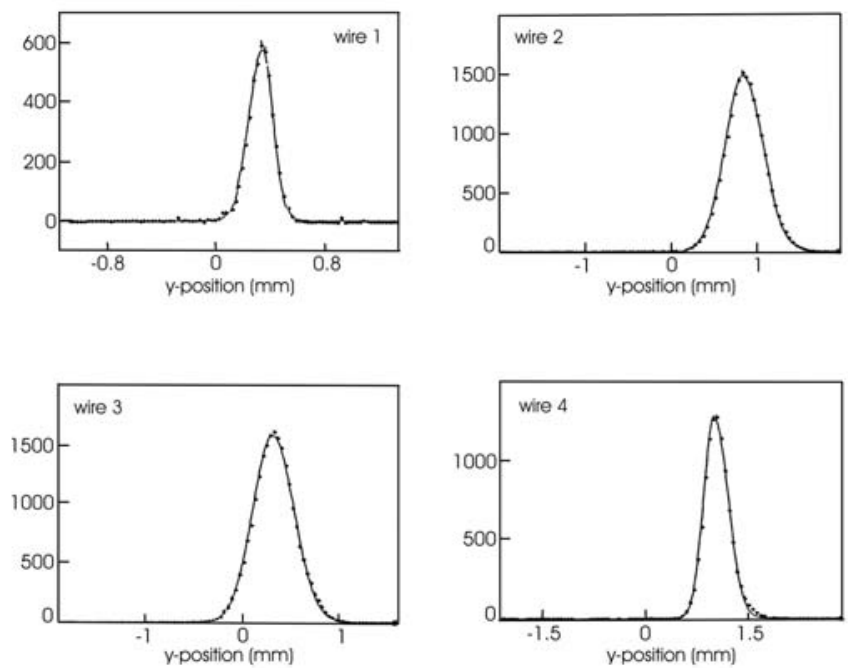

FIGURE 30. Raw $y$-plane data corresponding to the 4-dimensional emittance measurement summarized in Fig. 28.
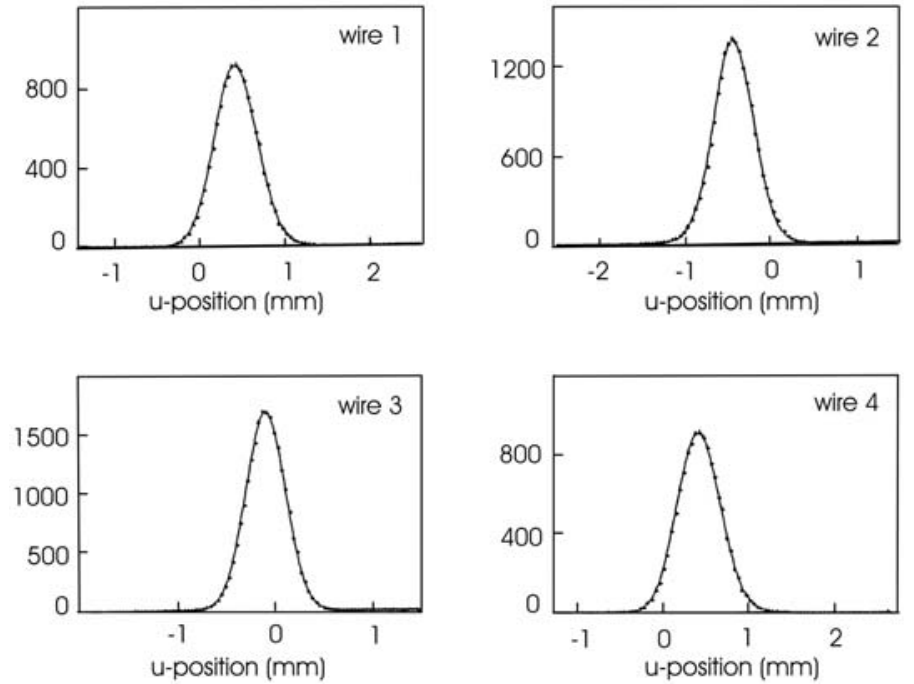

FIGURE 31. Raw $u$-plane data corresponding to the 4-dimensional emittance measurement summarized in Fig. 28. 